\title{
An efficient lattice Boltzmann method for compressible aerodynamics on D3Q19 lattice
}

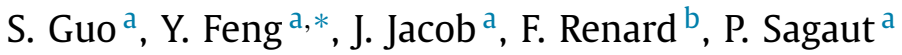 \\ a Aix Marseille Univ, CNRS, Centrale Marseille, M2P2 UMR 7340, 13451 Marseille, France \\ ${ }^{\mathrm{b}}$ CERFACS, Toulouse, France
}

\section{A R T I C L E I N F O}

\section{Article history:}

Received 24 July 2019

Received in revised form 27 April 2020

Accepted 13 May 2020

Available online 20 May 2020

\section{Keywords:}

D3Q19 lattice

High Mach

Hybrid thermal LBM

Shock sensor

Recursive regularized

\begin{abstract}
A B S T R A C T
An efficient lattice Boltzmann (LB) model relying on a hybrid recursive regularization (HRR) collision operator on D3Q19 stencil is proposed for the simulation of three-dimensional high-speed compressible flows in both subsonic and supersonic regimes. An improved thermal equilibrium distribution function on D3Q19 lattice is derived to reduce the complexity of correcting terms. A simple shock capturing scheme and an upwind biased discretization of correction terms are implemented for supersonic flows with shocks. Mass and momentum equations are recovered by an efficient streaming, collision and forcing process on D3Q19 lattice. Then a non-conservative formulation of the entropy evolution equation is used, that is solved using a finite volume method. The proposed method is assessed considering the simulation of i) 2D isentropic vortex convection, ii) 3D nonisothermal acoustic pulse, iii) 2D supersonic flow over a bump, iv) 3D shock explosion in a box, v) 2D vortex interaction with shock wave, vi) 2D laminar flows over a flat plate at Ma of $0.5,1.0$ and 1.5 .
\end{abstract}

\section{Introduction}

Accurate and efficient solutions of Euler and Navier-Stokes equations for fully compressible flows have been subject to intensive research in aerodynamics, combustion and aeroacoustic. They are very important for many fields of application including aerospace engineering, combustion-based propulsion and acoustic noise simulations.

As an alternative approach to simulate fluid flows based on the Boltzmann equation, the lattice Boltzmann method (LBM) has been proved to be very well suited for the simulation of nearly incompressible, athermal flows [1-6]. Motivated by its advantages for massively parallel computing as well as its ability to handle very complex geometries, there are significant research efforts devoted to extending LBM to thermal and subsonic to supersonic applications.

In achieving that goal of constructing LB models for fully compressible flows at higher Mach numbers, most of existing attempts are restricted to two-dimensional space and rely on finite volume/difference/element discretization [7-12]. The superiority of those LB models remains an open question compared with the gas kinetic schemes [13] and discrete velocities methods [11,14-16]. Focusing on the classical LB methods which are characterized by the canonical stream-and-collide algorithm that amounts to a Strang-splitting approach [17], it is required to consider high-order moments of density distribution functions to recover the macroscopic energy conservation equation for thermal lattice Boltzmann model [18-23],

\footnotetext{
* Corresponding author.

E-mail address: yongliang.feng@univ-amu.fr (Y. Feng).
} 
Table 1

List of stream-collide LB models for compressible aerodynamics. The abbreviations: fractional BGK(FBGK); entropic LB (ELB); ELB with shifted frame (ELB*); multiple relaxation time (MRT); fractional MRT (FMRT); single distribution function (SDF); double DF (DDF); regularized BGK (RBGK); recursive regularized BGK (RR); hybrid RR BGK (HRR); Rayleigh-Taylor (RT); boundary layer (BL); supersonic (sup.).

\begin{tabular}{|c|c|c|c|c|}
\hline Representative refs. & Discrete velocities & Collision model & Approach on energy & Test cases \\
\hline Alexander et al. [18] & D2Q13 & BGK & SDF & Couette flow \\
\hline Shan et al. [19] & D2Q17 etc. & BGK & SDF & \\
\hline Scagliarini et al. [20] & D2Q21/V37 & BGK & SDF & RT instability \\
\hline Philippi et al. [21] & D2V37 & BGK & SDF & \\
\hline Yu and Zhao [42] & D2V17 & BGK & athermal & sup. wedge \\
\hline Yan et al. [43] & D2V25 & BGK & SDF & sup. cylinder \\
\hline Prasianakis et al. [44] & D2Q9 & ELB & SDF & 1D shock tube \\
\hline Li et al. [45] & D2Q9 & MRT & DDF & acoustic \\
\hline Li et al. [46] & D2V37 & MRT & SDF & shock tube \\
\hline Coreixas et al [25] & D2V37 & $\mathrm{RR}$ & SDF & shock tube \\
\hline Mattila et al. [26] & D2V37 & $\mathrm{RR}$ & SDF & shock tube \\
\hline Saadat et al. [47] & D2Q9 & ELB* & DDF & shock vortex \\
\hline Feng et al. [40] & D2Q9 & HRR & hybrid & subsonic BL \\
\hline Chen et al. [48] & D3V40 & BGK & SDF & shear wave \\
\hline McNamara et al. [49] & D3V27 & FMRT & SDF & RB convection \\
\hline Nie et al. [34] & D3Q39 & FBGK & Hybrid & transonic airfoil \\
\hline Li et al. [50] & D3Q39 & RBGK & Hybrid & transonic airfoil \\
\hline Frapolli et al. [22] & D3Q343 & ELB & DDF & sup. airfoil \\
\hline Shan [51] & D3Q103 & BGK & SDF & \\
\hline Fares et al. [52,53] & D3Q39 & BGK & SDF & nozzle, airliner \\
\hline Latt et al. [54] & D3Q39 & BGK & DDF & sup. airfoil \\
\hline
\end{tabular}

usually relying on extended-neighbor lattice sets (D1Q7, D2Q37, D3Q343 etc.) leading to LB methods referred to as multispeed models [18-22,24-30]. This approach seems rather expensive for industrial applications due to the high number of discrete velocities and the increased complexity for the implementation of boundary conditions and local grid refinement. A brief overview of various 2D and 3D stream-and-collide-type LB models for compressible aerodynamics is reported in Table 1.

The thermal LB models based on the nearest-neighbor lattice sets for high Mach flows have attracted many research interests recently. A two-dimensional thermal lattice Boltzmann model in the manner of double-distribution function (DDF) was extended to high-subsonic flows using finite volume approach to improve numerical stability in [31]. A consistent two-distribution function thermal lattice Boltzmann with entropy stabilizer on compressible flows was proposed in [32]. In higher dimensions, a LB model using reduction of sound speed [33] and hybrid finite difference entropy equation was constructed for transonic and supersonic flows on D3Q39 lattice set [34,35], which is the closest one with 3D standard lattices (i.e. D3Q19, D3Q27). A three-dimensional DDF thermal lattice Boltzmann model for thermal flows was developed for simulation of shock explosion in a 3D box [36]. Recent breakthroughs have been made on regular lattices extending the hybrid Recursive Regularized scheme proposed in [37] to 2D compressible thermal flows [38,39] and then to 2D high Ma flows $[40,41]$. In the latter articles, the energy is taken into account via an entropy evolution equation written in quasi-linear form, that is solved using a stabilized finite volume approach.

Due to the three-dimensional intrinsic nature of realistic compressible flows, it is natural to seek a stable and efficient three-dimensional LB model which is able to simulate fully compressible flows using the minimum number of discrete velocities. In contrast to D3Q27 model, the D3Q19 model is computationally less expensive but has more flaws dealing with the breakdown of Galilean invariance, especially for high Mach number flows. The objective of the present paper is to further extend the approach proposed in [40] to 3D high-speed compressible flows, including flows with shock waves, on the D3Q19 lattice. We aim to construct an efficient LB model for 3D compressible flows using only 19 discrete velocities and 20 degrees of freedom per cell (19 distribution functions plus one thermodynamic scalar quantity for energy), which can be considered as minimum number of freedom per cell for compressible aerodynamics within the framework of LBM. To this end, both the expression of the equilibrium function and the Galilean-invariance-correction terms are revisited, along the stable discretization of the correction terms and entropy equation, as well as the appropriate shock capturing approach. The paper is organized as follows: In section 2, the proposed thermal lattice Botlzmann method with a new equilibrium distribution function on D3Q19 lattice is introduced. Section 3 presents theoretical analysis and implementation of entropy equation for energy conservation law. After that, the shock capturing approach and stable discretization of correction term in high Mach flows is analyzed and discussed in Sec. 4. Section 5 gives the details of the implementation of the present hybrid LB method and boundary conditions. Then, the results obtained considering six classical test cases are presented in Sec. 6 to assess the proposed method. Finally, section 7 draws conclusions and perspectives. 
Table 2

Discrete velocities and corresponding weights of D3Q27 and D3Q19.

\begin{tabular}{lllll}
\hline \multicolumn{3}{c}{ D3Q27 } & \multicolumn{3}{c}{ D3Q19 } \\
\hline$\left[c_{i \alpha}, w_{i}\right]$ & $(0,0,0)$ & $8 / 27$ & $(0,0,0)$ & $1 / 3$ \\
& $\operatorname{cyc}( \pm 1,0,0)$ & $2 / 27$ & $\operatorname{cyc}( \pm 1,0,0)$ & $1 / 18$ \\
& $\operatorname{cyc}( \pm 1, \pm 1,0)$ & $1 / 54$ & $\operatorname{cyc}( \pm 1, \pm 1,0)$ & $1 / 36$ \\
& $( \pm 1, \pm 1, \pm 1)$ & $1 / 216$ & & \\
\hline
\end{tabular}

\section{Hybrid thermal lattice Boltzmann method on D3Q19 lattice}

In the present hybrid thermal lattice Boltzmann method, mass and momentum equations are recovered by an efficient streaming, collision on D3Q19 lattice. Then a thermodynamic scalar quantity for energy is solved by using a finite volume method.

\subsection{Thermal lattice Boltzmannn model}

The thermal lattice Boltzmann model in this study aims at solving discrete velocity Boltzmann BGK equation in discrete physical space and time to obtain macroscopic density $\rho$ and velocity $u_{\alpha}$. The lattice Boltzmann equation with hybrid recursive regularization collision model is used in this study, that can be expressed as [40]

$$
f_{i}\left(x_{\alpha}+c_{i \alpha} \delta_{t}, t+\delta_{t}\right)=f_{i}^{e q}\left(x_{\alpha}, t\right)+\left(1-\frac{1}{\bar{\tau}}\right) \mathcal{R}\left(f_{i}^{n e q}\right)+\frac{\delta_{t}}{2} \psi_{i}\left(x_{\alpha}, t\right)
$$

In the LB algorithm, the macroscopic density $\rho$ and momentum $\rho u_{\alpha}$ are computed and updated as

$$
\begin{aligned}
& \rho=\sum_{i} f_{i}, \\
& \rho u_{\alpha}=\sum_{i} c_{i \alpha} f_{i}+\frac{\delta_{t}}{2} \sum_{i} c_{i \alpha} \psi_{i}
\end{aligned}
$$

In Eq. (1), $\bar{\tau}=\tau / \delta_{t}+1 / 2$ is non-dimensional relaxation time and $\mathcal{R}\left(f_{i}^{\text {neq }}\right)$ represents recursively regularized off-equilibrium part of the density distribution functions $f_{i}^{\text {neq }}=f_{i}-f_{i}^{e q}+\frac{\delta_{t}}{2} \psi_{i}$. The Galilean-invariance correction term $\psi_{i}$ is given in form of a force and $f_{i}{ }^{e q}$ is the thermodynamics equilibrium distribution function. The equilibrium distribution function and correction term on D3Q19 lattice are introduced and discussed in the following section.

\subsection{Improved equilibrium distribution function on D3Q19 lattice}

The setup of discrete equilibrium distribution functions and associated velocities is one among the key issues for the design of the compressible LB models. Following the approach discussed in $[19,40]$, the equilibrium distribution function $f^{e q}$ could be given in the third-order Grad-Hermite expansion of Maxwell-Boltzmann distribution as

$$
f_{i}^{e q, Q}=w_{i}\left[\rho+\frac{c_{i \alpha}}{c_{s}^{2}} \rho u_{\alpha}+\frac{\mathcal{H}_{i \alpha \beta}^{(2)}}{2 c_{s}^{4}} \mathcal{A}_{\alpha \beta}^{(0)}+\frac{\mathcal{H}_{i \alpha \beta \gamma}^{(3)}}{6 c_{s}^{6}} \mathcal{A}_{\alpha \beta \gamma}^{(0)}\right],
$$

where $w_{i}$ is the $i_{t h}$ weight coefficient associated to discrete velocity $c_{i \alpha}, c_{s}$ is lattice sound speed and the discrete Hermite polynomials are given as $\mathcal{H}_{i \alpha \beta}^{(2)}=c_{i \alpha} c_{i \beta}-c_{s}^{2}, \mathcal{H}_{i \alpha \beta \gamma}^{(3)}=c_{i \alpha} c_{i \beta} c_{i \gamma}-c_{s}^{2}\left[c_{i} \delta\right]_{\alpha \beta \gamma}$ and $\left[c_{i} \delta\right]_{\alpha \beta \gamma}=c_{i \alpha} \delta_{\beta \gamma}+c_{i \beta} \delta_{\alpha \gamma}+c_{i \gamma} \delta_{\alpha \beta}$. The second and third-order terms in the equilibrium distribution function are $\mathcal{A}_{\alpha \beta}^{(0)}=\rho u_{\alpha} u_{\beta}+\rho c_{s}^{2}(\theta-1) \delta_{\alpha \beta}, \mathcal{A}_{\alpha \beta \gamma}^{(0)}=$ $\rho u_{\alpha} u_{\beta} u_{\gamma}+\rho c_{s}^{2}(\theta-1)[u \delta]_{\alpha \beta \gamma}$ with $[u \delta]_{\alpha \beta \gamma}=u_{\alpha} \delta_{\beta \gamma}+u_{\beta} \delta_{\alpha \gamma}+u_{\gamma} \delta_{\alpha \beta} . \theta$ is defined according to temperature $T$ as $\theta=R T / c_{s}^{2}$.

The parameter $Q$ denotes the number of discrete velocities. For the three-dimensional lattices, there are two common discrete velocity models, namely the nineteen-velocity model (D3Q19) and the twenty-seven-velocity model (D3Q27) as shown in Table 2, which are associated to the equilibrium functions $f_{i}^{e q, 19}$ and $f_{i}^{e q, 27}$, respectively.

Considering the equilibrium distribution function of Eq. (3), the deviation terms on the third-order moment due to the defect of symmetry can be calculated as follows

$$
\Psi_{\alpha \beta \gamma}=\Pi_{\alpha \beta \gamma}^{e q, M}-\Pi_{\alpha \beta \gamma}^{e q, Q}
$$

where $\Psi_{\alpha \beta \gamma}$ denotes deviation terms on the third-order moment. $\Pi_{\alpha \beta \gamma}^{e q, M}$ and $\Pi_{\alpha \beta \gamma}^{e q, Q}$ are respectively the third-order moment computed by exact Maxwellian distribution and by Q-site velocities discrete equilibrium distribution function as follows 
Table 3

The third-order moment of 3D lattice models.

\begin{tabular}{lllll}
\hline$\Pi_{\alpha \beta \gamma}^{e q}$ & $\mathrm{D} 3 \mathrm{Q} 27(3)$ & $\mathrm{D} 3 \mathrm{Q} 19(3)$ & D3Q19r (8) & Maxwellian (7) \\
\hline$\Pi_{x x x}^{e q}$ & $\rho u_{x}$ & $\rho u_{x}$ & $\rho u_{x}$ & $\rho u_{x} u_{x} u_{x}+3 p u_{x}$ \\
$\Psi_{x x x}$ & $\rho u_{x}\left(\theta-1+u_{x}^{2}\right)$ & $\rho u_{x}\left(\theta-1+u_{x}^{2}\right)$ & $\rho u_{x}\left(\theta-1+u_{x}^{2}\right)$ & 0 \\
& & & \\
$\Pi_{x x y}^{e q}$ & $\rho u_{x} u_{x} u_{y}+p u_{y}$ & $\frac{\theta+1}{2} \rho c_{s}^{2} u_{y}+\rho u_{y}\left(u_{x}^{2}-\frac{u_{z}^{2}}{2}\right)$ & $\rho u_{x} u_{x} u_{y}+p u_{y}$ & $\rho u_{x} u_{x} u_{y}+p u_{y}$ \\
$\Psi_{x x y}$ & 0 & $\rho u_{y}\left(\frac{\theta-1}{6}+\frac{u_{z}^{2}}{2}\right)$ & 0 & 0 \\
$\Pi_{x y z}^{e q}$ & $\rho u_{x} u_{y} u_{z}$ & 0 & 0 & \\
$\Psi_{x y z}$ & 0 & $\rho u_{x} u_{y} u_{z}$ & $\rho u_{x} u_{y} u_{z}$ & 0 \\
\hline
\end{tabular}

$$
\begin{aligned}
& \Pi_{\alpha \beta \gamma}^{e q, M}=\int c_{\alpha} c_{\beta} c_{\gamma} f^{e q, M} \mathrm{~d}^{3} c=p[u \delta]_{\alpha \beta \gamma}+\rho u_{\alpha} u_{\beta} u_{\gamma}, \\
& \Pi_{\alpha \beta \gamma}^{e q, Q}=\sum_{i=0}^{Q-1} c_{i \alpha} c_{i \beta} c_{i \gamma} f_{i}^{e q, Q}
\end{aligned}
$$

where the pressure $p=\rho c_{s}^{2} \theta$ satisfies the equation of state of perfect gas. $f^{e q, M}$ is Maxwell-Boltzmann distribution function, which is written as

$$
f^{e q, M}=\rho\left(\frac{1}{2 \pi R T}\right)^{\frac{3}{2}} \exp \left[-\frac{\left(\xi_{\alpha}-u_{\alpha}\right)^{2}}{2 R T}\right]
$$

where $R$ is the gas constant. The third-order moments $\Pi_{\alpha \beta \gamma}^{e q, 27}$ and $\Pi_{\alpha \beta \gamma}^{e q, 19}$ computed using D3Q27 and D3Q19 lattices following Eq. (3) and $\Pi_{\alpha \beta \gamma}^{e q, M}$ computed using the exact Maxwellian equilibrium distribution functions as well as the associated deviation terms of $\Psi_{\alpha \beta \gamma}$ are summarized in Table 3. It is observed from the table that only $\Psi_{x x x}, \Psi_{y y y}, \Psi_{z z z}$ is non-zero in D3Q27 lattice set, which render the correction term much simpler compared with the one obtained on the D3Q19 lattice [40]. Considering the symmetry of Gaussian-Hermite moment [55,56], the following non-isothermal equilibrium distribution function on D3Q19 lattice (denoted as D3Q19r) is introduced to reduce the defect of the third-order moment

$$
\begin{aligned}
& f_{i}^{e q, 19 r}=w_{i}\left[\rho+\frac{c_{i \alpha}}{c_{s}^{2}} \rho u_{\alpha}+\frac{\mathcal{H}_{i \alpha \beta}^{(2)}}{2 c_{s}^{4}} \mathcal{A}_{\alpha \beta}^{(0)}+\frac{[\mathcal{H} \mathcal{A}]_{i, Q 19 r}^{(3,0)}}{6 c_{s}^{6}}\right] \\
& =w_{i}\left\{\rho+\frac{c_{i \alpha} \rho u_{\alpha}}{c_{s}^{2}}+\frac{A_{\alpha \beta}^{(0)} \mathcal{H}_{i \alpha \beta}^{(2)}}{2 c_{s}^{4}}+\frac{1}{6 c_{s}^{6}}[\right. \\
& 3\left(\mathcal{H}_{i, x x y}^{(3)}+\mathcal{H}_{i, y z z}^{(3)}\right)\left(A_{x x y}^{(0)}+A_{y z z}^{(0)}\right)+\left(\mathcal{H}_{i, x x y}^{(3)}-\mathcal{H}_{i, y z z}^{(3)}\right)\left(A_{x x y}^{(0)}-A_{y z z}^{(0)}\right) \\
& +3\left(\mathcal{H}_{i, x z z}^{(3)}+\mathcal{H}_{i, x y y}^{(3)}\right)\left(A_{x z z}^{(0)}+A_{x y y}^{(0)}\right)+\left(\mathcal{H}_{i, x z z}^{(3)}-\mathcal{H}_{i, x y y}^{(3)}\right)\left(A_{x z z}^{(0)}-A_{x y y}^{(0)}\right) \\
& \left.\left.+3\left(\mathcal{H}_{i, y y z}^{(3)}+\mathcal{H}_{i, x x z}^{(3)}\right)\left(A_{y y z}^{(0)}+A_{x x z}^{(0)}\right)+\left(\mathcal{H}_{i, y y z}^{(3)}-\mathcal{H}_{i, x x z}^{(3)}\right)\left(A_{y y z}^{(0)}-A_{x x z}^{(0)}\right)\right]\right\}
\end{aligned}
$$

The third-order moments $\Pi_{\alpha \beta \gamma}^{e q, 19 r}$ stemming from the new equilibrium function for the D3Q19 lattice given by Eq. (8) are also summarized in Table 3. As shown in the table, the third-order moment terms related to $c_{i x} c_{i x} c_{i x}$ and $c_{i x} c_{i y} c_{i z}$ remain the same as for the original $f_{i}^{e q, 19}$ equilibrium function when considering $f_{i}^{e q, 19 r}$, but the exact terms for $c_{i x} c_{i y} c_{i y}$ are fully recovered by the revised equilibrium distribution function. The deviation terms $\Psi_{x y z}$ and $\Psi_{x x x}$ on $c_{i x} c_{i y} c_{i z}$ and $c_{i x} c_{i x} c_{i x}$ are balanced by introducing a correcting term $\psi_{i}$, which can be applied as a forcing term in lattice Boltzmann BGK equation (1).

$$
\psi_{i}=-w_{i} \frac{\mathcal{H}_{i \alpha \beta}^{(2)}}{2 c_{s}^{4}} \frac{\partial}{\partial x_{\gamma}} \Psi_{\alpha \beta \gamma}, \quad \sum_{i} c_{i \alpha} c_{i \beta} \psi_{i}=-\frac{\partial}{\partial x_{\gamma}} \Psi_{\alpha \beta \gamma}
$$

The correction term $\psi_{i}$ on D3Q19 lattice is given in the following simple form: 


$$
\begin{aligned}
\psi_{i}=\frac{w_{i}}{2 c_{s}^{4}}\left\{\mathcal{H}_{i x x}^{(2)} \frac{\partial}{\partial x}\left[\rho u_{x}\left(1-\theta-u_{x}^{2}\right)\right]-\mathcal{H}_{i y z}^{(2)} \frac{\partial}{\partial x}\left(\rho u_{x} u_{y} u_{z}\right)\right. \\
+\mathcal{H}_{i y y}^{(2)} \frac{\partial}{\partial y}\left[\rho u_{y}\left(1-\theta-u_{y}^{2}\right)\right]-\mathcal{H}_{i x z}^{(2)} \frac{\partial}{\partial y}\left(\rho u_{x} u_{y} u_{z}\right) \\
\left.+\mathcal{H}_{i z z}^{(2)} \frac{\partial}{\partial z}\left[\rho u_{z}\left(1-\theta-u_{z}^{2}\right)\right]-\mathcal{H}_{i x y}^{(2)} \frac{\partial}{\partial z}\left(\rho u_{x} u_{y} u_{z}\right)\right\}
\end{aligned}
$$

Following the Chapman-Enskog multiscale technique (see Appendix A), the mass and momentum conservation equations can be recovered as follows

$$
\begin{aligned}
& \frac{\partial \rho}{\partial t}+\frac{\partial}{\partial x_{\alpha}}\left(\rho u_{\alpha}\right)=0, \\
& \frac{\partial}{\partial t}\left(\rho u_{\alpha}\right)+\frac{\partial}{\partial x_{\beta}}\left(\rho u_{\alpha} u_{\beta}+p \delta_{\alpha \beta}\right)=\frac{\partial}{\partial x_{\beta}}\left(\Pi_{\alpha \beta}\right),
\end{aligned}
$$

where $\Pi_{\alpha \beta}$ is stress tensor,

$$
\Pi_{\alpha \beta}=\mu\left[\frac{\partial u_{\beta}}{\partial x_{\alpha}}+\frac{\partial u_{\alpha}}{\partial x_{\beta}}-\frac{2}{3} \frac{\partial u_{\gamma}}{\partial x_{\gamma}} \delta_{\alpha \beta}\right]
$$

$\mu$ is dynamic viscosity, which is related with non-dimensional relaxation time through $\mu=p(\bar{\tau}-0.5) \delta_{t}$ in the present thermal lattice Boltzmann model.

\subsection{Associated recursive regularization on D3Q19 lattice}

The recursively regularized off-equilibrium distribution function on D2Q9 or original D3Q19 model as given in Ref. [40] is expressed as follows

$$
\mathcal{R}\left(f_{i}^{n e q}\right)=w_{i}\left[\frac{\mathcal{H}_{i \alpha \beta}^{(2)}}{2 c_{s}^{4}} \mathcal{A}_{\alpha \beta}^{(1)}+\frac{\mathcal{H}_{i \alpha \beta \gamma}^{(3)}}{6 c_{s}^{6}} \mathcal{A}_{\alpha \beta \gamma}^{(1)}\right]
$$

where $\mathcal{A}_{\alpha \beta}^{(1)}=\sum_{i} c_{i \alpha} c_{i \beta} f_{i}^{\text {neq }}$ and $\mathcal{A}_{\alpha \beta \gamma}^{(1)} \approx u_{\alpha} \mathcal{A}_{\beta \gamma}^{(1)}+u_{\beta} \mathcal{A}_{\gamma \alpha}^{(1)}+u_{\gamma} \mathcal{A}_{\alpha \beta}^{(1)}$. Considering the same symmetry feature of coefficient of Hermite polynomials for off-equilibrium, the associated off-equilibrium on improved D3Q19 model is now recursively regularized as follows

$$
\begin{aligned}
& \mathcal{R}\left(f_{i}^{n e q}\right)=w_{i}\left\{\frac{A_{\alpha \beta}^{(1)} \mathcal{H}_{i \alpha \beta}^{(2)}}{2 c_{s}^{4}}+\frac{1}{6 c_{s}^{6}}[\right. \\
& 3\left(\mathcal{H}_{i, x x y}^{(3)}+\mathcal{H}_{i, y z z}^{(3)}\right)\left(A_{x x y}^{(1)}+A_{y z z}^{(1)}\right)+\left(\mathcal{H}_{i, x x y}^{(3)}-\mathcal{H}_{i, y z z}^{(3)}\right)\left(A_{x x y}^{(1)}-A_{y z z}^{(1)}\right) \\
& +3\left(\mathcal{H}_{i, x z z}^{(3)}+\mathcal{H}_{i, x y y}^{(3)}\right)\left(A_{x z z}^{(1)}+A_{x y y}^{(1)}\right)+\left(\mathcal{H}_{i, x z z}^{(3)}-\mathcal{H}_{i, x y y}^{(3)}\right)\left(A_{x z z}^{(1)}-A_{x y y}^{(1)}\right) \\
& \left.\left.+3\left(\mathcal{H}_{i, y y z}^{(3)}+\mathcal{H}_{i, x x z}^{(3)}\right)\left(A_{y y z}^{(1)}+A_{x x z}^{(1)}\right)+\left(\mathcal{H}_{i, y y z}^{(3)}-\mathcal{H}_{i, x x z}^{(3)}\right)\left(A_{y y z}^{(1)}-A_{x x z}^{(1)}\right)\right]\right\}
\end{aligned}
$$

It is worth noting that the coefficient of the second viscosity being $-2 / 3$ is naturally recovered by D3Q19 lattice model in contrast to D2Q9 model [40]. In the same manner used in [37,40], a parameterized hybrid recursive regularized procedure is adopted to suppress non-hydrodynamic modes by using $\mathcal{A}_{\alpha \beta}^{(1, \mathrm{HRR})}=\sigma \mathcal{A}_{\alpha \beta}^{(1)}+(1-\sigma) \mathcal{A}_{\alpha \beta}^{(1, \mathrm{FD})} . \sigma \in[0,1]$ is an arbitrary weighting coefficient. $\mathcal{A}_{\alpha \beta}^{(1, \mathrm{FD})}$ is estimated by its Chapman-Enskog solution which is approximated by a second-order finitedifference scheme [40].

\section{Entropy based energy conservation equation}

In the present study, the thermodynamic quantity for energy conservation is solved by a finite volume method. The numerical stability of the solution is intrinsically tied to the control of the discrete energy of the solution, which must remain bounded. As discussed in the case of Navier-Stokes solutions, e.g. [57], the solution should preserve the second law of thermodynamic for entropy evolution. Therefore, the explicit control of the entropy appears as a natural way to enforce both numerical stability and physical evolution of the entropy at the same time. This lead several groups to work on the entropic variables to solve both Euler and Navier-Stokes equations, e.g. [58], or to design numerical method that preserve entropy in smooth flows [59-66], or to derive new LBM schemes based on the preservation of an entropic principle for manufactured entropy variable [22,24,67-69].

As a matter of fact, the mass conservation is not explicitly and strictly guaranteed in LB methods, something that can be shown considering the Chapman-Enskog expansion [70]. This lack of exact mass conservation in LBM must be taken into 
account when designing an hybrid method. For instance, an error term $\epsilon_{\rho}$ implicitly exists in mass conservation equation recovered by LBM

$$
\frac{\partial \rho}{\partial t}+\frac{\partial}{\partial x_{\alpha}}\left(\rho u_{\alpha}\right)=\epsilon_{\rho}
$$

where $\epsilon_{\rho}$ scales as $\mathcal{O}\left(\mathrm{Kn}^{2} \frac{\partial^{3}\left(\rho u_{\alpha}\right)}{\partial x_{\alpha} \partial x_{\beta} \partial x_{\beta}}\right)$ by considering third-order terms in the Chapman-Enskog expansion [70]. Here, the Knudsen number $\mathrm{Kn}$ is assumed to be a small parameter, which is true for the class of flows considered in the present paper. It is worth keeping in mind that a hierarchy of hydrodynamic models can be derived from the Boltzmann equation thanks to the Chapman-Enskog expansion (by increasing order in terms of small parameter expansion: Euler, Navier-Stokes, Burnett, super-Burnett...) showing that the departure from Euler and Navier-Stokes equations is a physical phenomenon.

In order to analyze the impact of $\epsilon_{\rho}$ on the entropy evolution, a density $\rho_{c}$ that satisfies the exact mass conservation is introduced, i.e.

$$
\frac{\partial \rho_{c}}{\partial t}+\frac{\partial}{\partial x_{\alpha}}\left(\rho_{c} u_{\alpha}\right)=0
$$

Now omitting viscous production terms for the sake of simplicity without restricting the generality of the demonstration, and starting from non-conservative form of the entropy equation

$$
\frac{\partial s}{\partial t}+u_{\alpha} \frac{\partial s}{\partial x_{\alpha}}=0
$$

the following usual conservative form of entropy evolution equation is obtained by combining Eqs. (16) and (17):

$$
\frac{\partial \rho_{c} s}{\partial t}+\frac{\partial \rho_{c} u_{\alpha} s}{\partial x_{\alpha}}=0
$$

where no spurious source term appears, thanks to the exact mass conservation.

This equation must be modified to account for the $\epsilon_{\rho}$ term. Starting from the conservative form of entropy equation

$$
\frac{\partial \rho s}{\partial t}+\frac{\partial \rho u_{\alpha} s}{\partial x_{\alpha}}=0
$$

and accounting for Eqs. (15), (16) and (19), the following conservative form of the entropy equation is obtained

$$
\frac{\partial \rho_{c} s}{\partial t}+\frac{\partial \rho_{c} u_{\alpha} s}{\partial x_{\alpha}}=-\frac{\rho_{c}}{\rho} \epsilon_{\rho} s .
$$

Consequently, the conservative form of entropy-based energy conservation could induce spurious entropy source, while this term is not present when the non-conservative formulation is used. It is worth noting that the same conclusion holds when considering the use of other thermodynamical quantities such as internal energy or total energy. A numerical validation of this theoretical analysis for non-conservative form entropy equation is carried out in Sec. 6.1. Therefore, a non-conservative form of entropy equation is used in the paper.

$$
\frac{\partial s}{\partial t}+u_{\alpha} \frac{\partial s}{\partial x_{\alpha}}=\frac{1}{\rho T} \frac{\partial}{\partial x_{\alpha}}\left(\lambda \frac{\partial T}{\partial x_{\alpha}}\right)+\frac{1}{\rho T} \Pi_{\alpha \beta} \frac{\partial u_{\alpha}}{\partial x_{\beta}},
$$

where $s=c_{v} \ln \left(p / \rho^{\gamma}\right)$ is the entropy with $c_{v}$ being specific heat capacity at constant volume and specific heat ratio $\gamma$. $\lambda$ is heat conductivity.

An explicit Euler scheme is adopted as temporal integration. The convective flux is constructed using third-order MUSCL scheme [71] and van Albada limiter [72], while the classical second-order central difference scheme is adopted for the diffusion term and term of viscous dissipation.

\section{Shock capturing technique and improved discretization of correction term}

\subsection{Shock capturing technique}

The computation of flow containing shock waves is an extremely difficult task because such flows result in sharp, discontinuous changes in flow variables such as pressure, temperature, density, and velocity across the shock. In order to capture the shock and increase numerical stability, the shock sensor and associated artificial viscosity used in Jameson-SchmidtTurkel (JST) scheme [73] is adopted in the present LB model to detect the shock wave for non-smooth compressible flows, which is given as

$$
\varepsilon_{\alpha}=\kappa\left|\frac{p_{i-1}-2 p_{i}+p_{i+1}}{p_{i-1}+2 p_{i}+p_{i+1}}\right|
$$


where $i$ is the index of Cartesian grid. $\kappa$ is a free parameter introduced to tune the artificial viscosity. The value of $\kappa$ is set to unity in all the simulations of this study. Accordingly, an effective relaxation time can be summed as

$$
\tau_{e}=\frac{\mu}{p}+\max \left[\varepsilon_{x}, \varepsilon_{y}, \varepsilon_{z}\right] \delta_{t}
$$

Then the non-dimensional relaxation time $\bar{\tau}=\tau_{e} / \delta_{t}+0.5$ is actually employed in the following test cases with shocks. It is worth noting that the variables, e.g. density $\rho$, entropy $s$ can be alternatively used in Eq. (22).

\subsection{Upwind-biased discretization of correction term}

The discretization of corrections term in Eq. (10) requires specific treatment in supersonic flows with respect to the subsonic flow case to ensure numerical stability, considering that the truncation error of its finite difference approximation may potentially violent Galilean invariant. The correction term is derived from third-order moment of equilibrium distribution function, which involves convective fluxes of the energy equation. Although the energy equation is not directly solved by the LB approach, the discrete energy of the LB solution obeys an evolution equation that can be derived from the LBM scheme, exactly as done for the discrete energy of Navier-Stokes computational solution, e.g., [74,75,61,62,57,76-80,63]. As a consequence, the thermal and kinetic energy oscillations can implicitly weaken the numeral stability. Therefore, a dissipative term is introduced in the momentum equation in order to prevent spurious growth of the discrete energy of the solution, which appears as a sink term in the discrete energy equation. The detailed Chapman-Enskog analysis of the energy equation is presented in Appendix A.

In order to improve the numerical stability by controlling the discrete energy of the solution, a low-dissipative secondorder upwind scheme is adopted in this study to discretized the correction term in the momentum equation, e.g., for $u>0$

$$
\frac{\partial \Psi}{\partial x}=\frac{1}{4 \Delta x}\left(\Psi_{i-2}-5 \Psi_{i-1}+3 \Psi_{i}+\Psi_{i+1}\right)
$$

where a half central difference and a half second-order upwind difference is used in this upwind scheme to approximate the partial difference operator in the correction term. It is in contrast to isotropic central discretization in the compressible LB model for subsonic flows [40].

\section{Implementation of the method}

The full procedure of the hybrid LB method for compressible aerodynamics is summarized as follows

1. Initialize the macroscopic variables $\rho, u_{\alpha}, T, s$ as well as density distribution function and correction term $\psi_{i}$.

2. Implement boundary treatment for macroscopic variables and density distribution. i) The macroscopic velocities $\rho_{B}, \boldsymbol{u}_{B}$, $T_{B}$ and $p_{B}$ on the boundary nodes are directly computed for the planar boundaries or estimated from their neighbor fluid nodes using Shepard's Inverse Distance Weighting (IDW) method for curved boundaries, following boundary conditions commonly used in classical Navier-Stokes solvers. ii) the density distribution function is computed by a finite difference reconstruction approach $[81,40]$. iii) The boundary condition for entropy equation, entropy $s_{B}$, is calculated using $\rho_{B}$ and $p_{B}$ by thermodynamic closure. Implementation details are the same as in [40] and will not be repeated here.

3. Perform collision and streaming procedure by Eq. (1) at the $n+1$ time step.

4. Update density $\rho$ and velocity $u_{\alpha}$ at the $\mathrm{n}+1$ time step by using Eq. (2).

5. Solve entropy equation (21) by the finite volume method and update $T$ and $p$ of the $n+1$ time step by using thermodynamic closure.

\section{Validation: numerical results and discussion}

The proposed method is assessed considering six cases dealing with compressible subsonic to supersonic flows:

1. 2D isentropic vortex convection,

2. 3D non-isothermal acoustic pulse,

3. 2D supersonic flow over a bump,

4. 3D shock explosion in a box,

5. 2D vortex interaction with shock wave,

6. 2D laminar flow with $\mathrm{Ma}=(0.5,1.0,1.5)$ over a flat plate.

In these simulations, the inviscid flows are treated as quasi-inviscid, with a very small non-dimensional viscosity $\mu=10^{-15}$. The value of $\sigma$ plays a role of hyper-viscosity in the HRR-LBM model and the hyper-viscosity decreases by increasing $\sigma$, which was illustrated and investigated in [37,40]. As a matter of fact, the global second-order accuracy of the method with $\sigma=0.5$ is well confirmed in the case of isentropic vortex convection in Sec. 6. Thus, the recommended minimum value $\sigma$ is $\sigma=0.5$ in the in-viscid compressible flows, $\sigma=0.7$ in the viscous supersonic flows, and $\sigma=0.9$ in the viscous high 


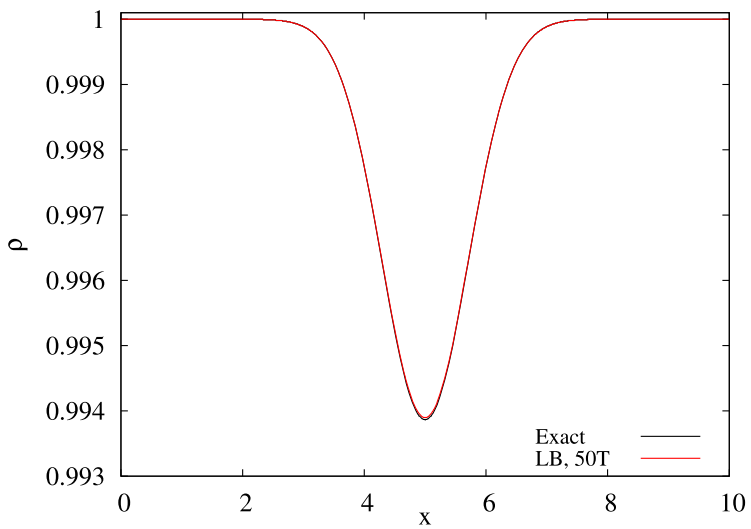

(a) Density

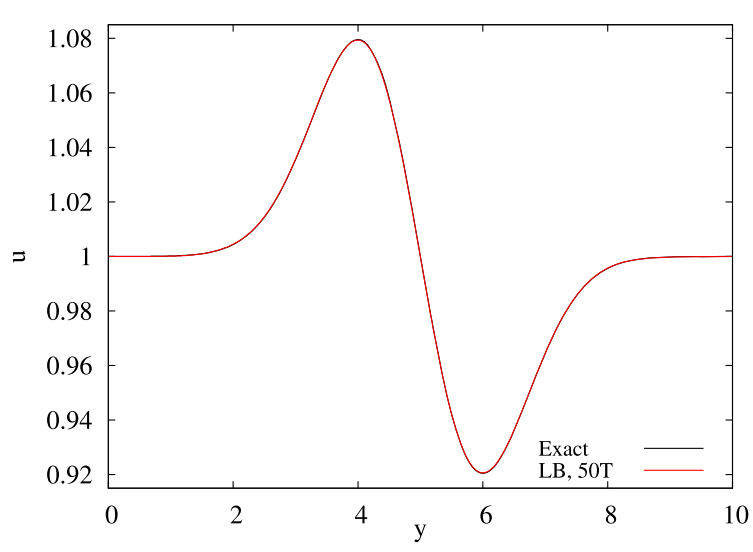

(b) Velocity

Fig. 1. The distributions of density and velocity on the mid-line at $t=50 T(\Delta x=0.025)$. (For interpretation of the colors in the figure(s), the reader is referred to the web version of this article.)

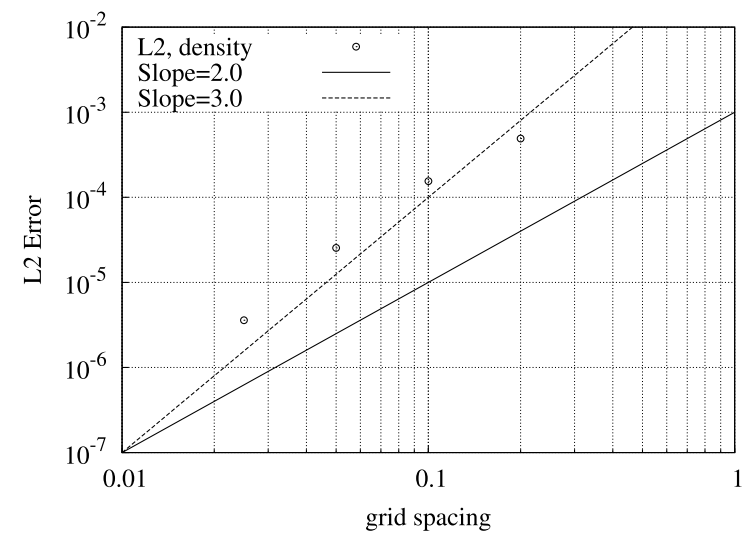

Fig. 2. Convergence rate study of the proposed LB. The relative error is computed through $L_{2}$ error on density at $t=50 T$.

subsonic flows. All of the 2D and 3D computational examples presented in the next section were obtained within the D3Q19 framework in the ProLB solver [82].

\subsection{Isentropic vortex convection}

The problem of isentropic vortex convection is first considered to assess the proposed HRR-LB method and to investigate the influence of the formulation of the entropy equation. The size of the computational domain is $[0,10] \times[0,10]$. The uniform free-stream parameters are $\rho_{\infty}=1, u_{\infty}=1.0, v_{\infty}=0, p_{\infty}=1, M a_{\infty}=0.84515$. At the initial time, the following disturbance is added to the above free-stream:

$$
\begin{aligned}
& \rho=\left[1-\frac{(\gamma-1) b^{2}}{8 \gamma \pi^{2}} e^{1-r^{2}}\right]^{\frac{1}{\gamma-1}}, p=\rho^{\gamma}, \\
& u=u_{\infty}-\frac{b}{2 \pi} e^{\frac{1}{2}\left(1-r^{2}\right)}\left(y-y_{c}\right), \\
& v=v_{\infty}+\frac{b}{2 \pi} e^{\frac{1}{2}\left(1-r^{2}\right)}\left(x-x_{c}\right),
\end{aligned}
$$

where $b=0.5, x_{c}=5, y_{c}=5$ and $r=\left[\left(x-x_{c}\right)^{2}+\left(y-y_{c}\right)^{2}\right]^{1 / 2}$. In this simulation, the HRR weighting parameter is $\sigma=0.5$. Fig. 1 shows the distributions of density and velocity along the symmetry line of the domain after 50 flow-through-times, i.e. $t=50 T$. These distributions are obtained using a grid size equal to $\Delta x=0.025$. From the figure, it can be seen that present results match very well with the analytical solution.

In order to study the global spatial accuracy of the present method, different mesh sizes are now considered, i.e. $\Delta x=$ $0.05,0.1,0.2$. Fig. 2 displays the convergence rate of the $L_{2}$-norm of the error of the proposed LB method. For the fine grid 


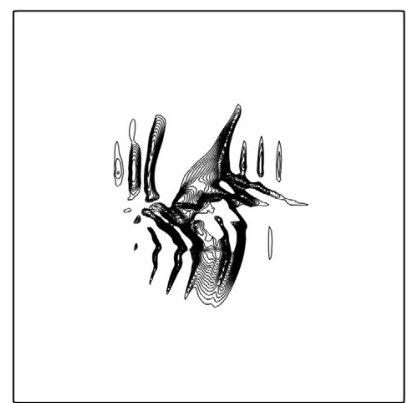

(a) $\rho s, t=1 T$

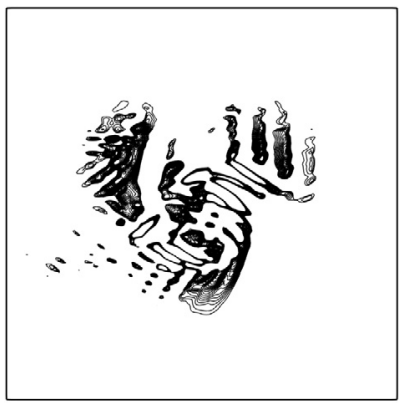

(d) $\rho s, t=2 T$

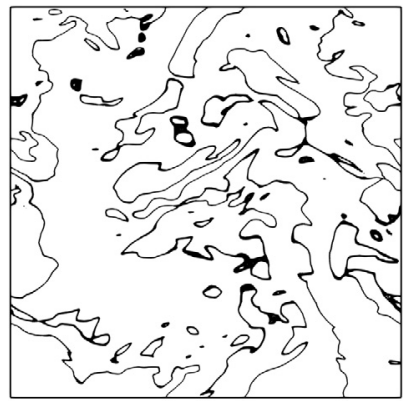

(g) $\rho s, t=50 T$

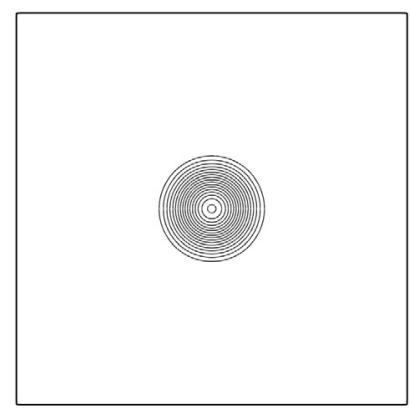

(b) $\rho_{c} s, t=1 T$

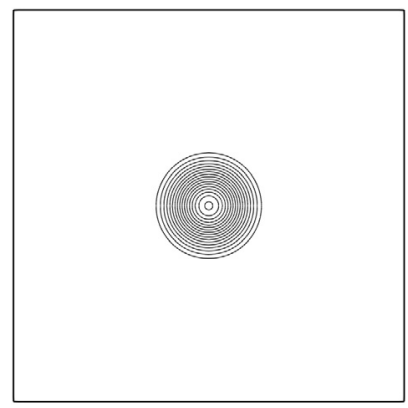

(e) $\rho_{c} s, t=2 T$

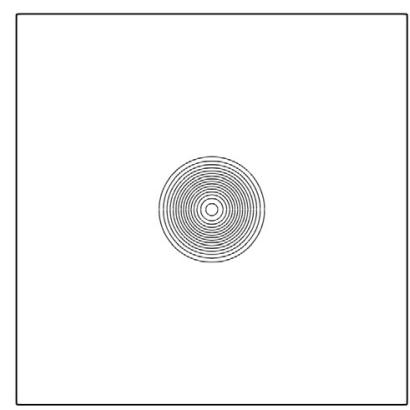

(h) $\rho_{c} s, t=50 T$

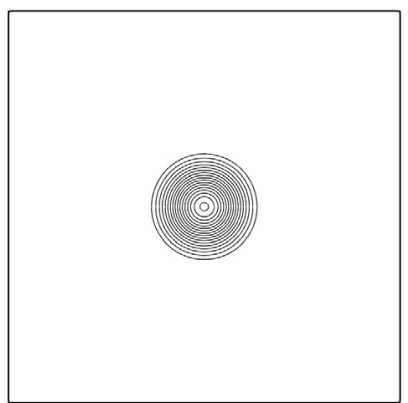

(c) $s, t=1 T$

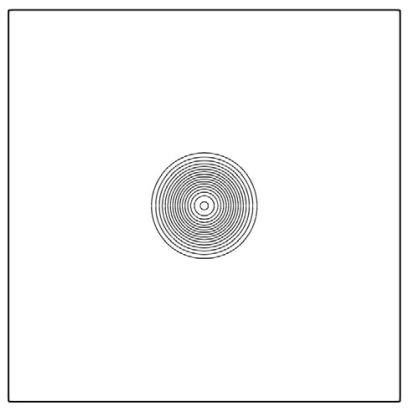

(f) $s, t=2 T$

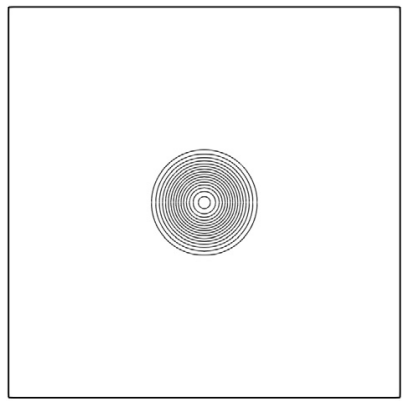

(i) $s, t=50 T$

Fig. 3. The density contours (from 0.993 to 0.999 with 20 levels). Left: results obtained considering the conservative form of the entropy equation; middle: results obtained using the present LB method with a conservative entropy equation, but in which an auxiliary density field computed by solving the exact mass conservation equation with a finite volume solver is used; right: present LB method with non-conservative entropy equation.

resolutions, it is observed that the present method exhibits a super-convergence property with a slope close to 3, as already reported for some LB methods [83]. These results assess the accuracy of present compressible HRR-LBM method equipped with 3rd-order MUSCL scheme for the entropy convection term.

As mentioned in section 3, the conservative form of entropy-based energy conservation equation could induce erroneous entropy sources. This point will now be illustrated by adequate numerical experiments. More precisely, three methods will be compared:

- HRR-LBM supplemented by an equation for $\rho s$ written in conservative form

- HRR-LBM supplemented by an auxiliary equation for $\rho_{c} s$ written in conservative form, where the "exact" density $\rho_{c}$ is computed solving an additional equation by a classical finite volume method. This method is introduced here only to assess the theoretical analysis given above, and is not proposed as a regular method for applications.

- HRR-LBM supplemented by an equation for $s$ written in non-conservative form

Results obtained with these three approaches are displayed in Fig. 3. It can be seen from these results that the spurious entropy production corrupts the evolution of density very quickly. However, both the conservative entropy with corrected density and non-conservative entropy exhibit very good solutions and numerical stability. This point is further validated looking at the time history of the entropy field displayed in Fig. 4. It is observed that the non-conservative form of en- 


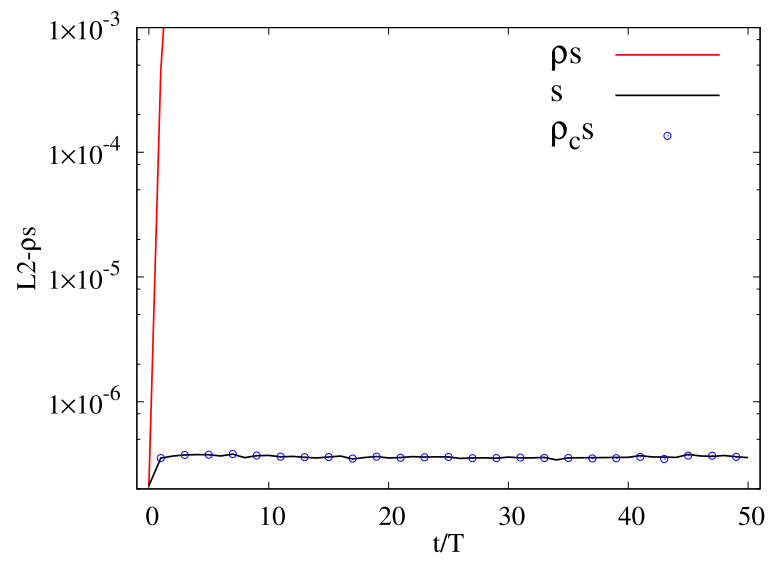

Fig. 4. Time history of the entropy obtained by the different forms of entropy equation.

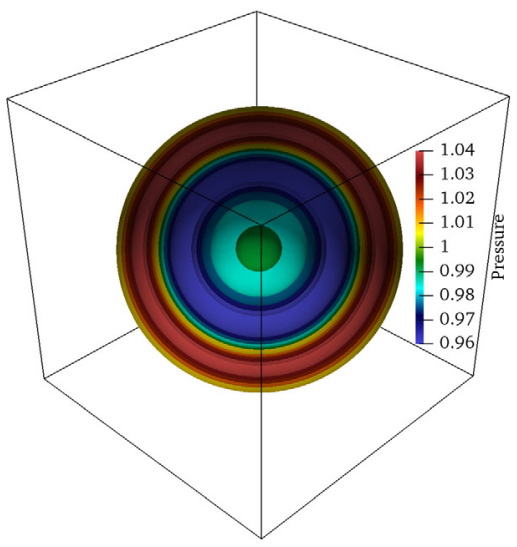

(a) pressure

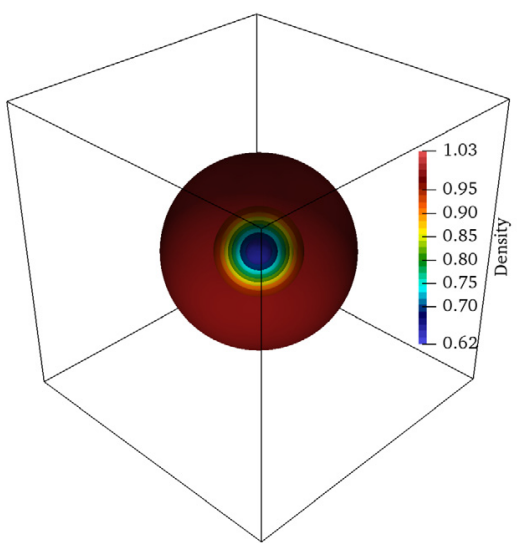

(c) density

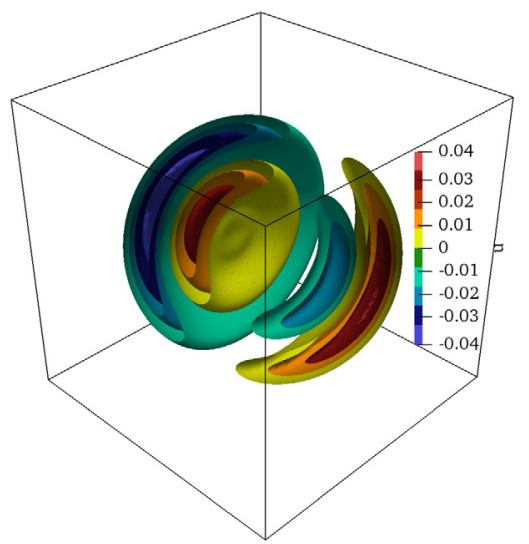

(b) velocity

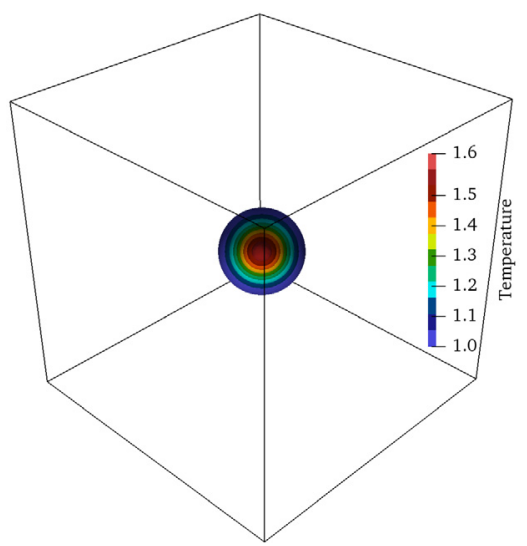

(d) temperature

Fig. 5. The pressure, u-velocity, density and temperature fields at time $t_{\text {end }}=1.0$ obtained using pure recursive-regularization collision model (parameter $\sigma=1$ ) on grid resolution of $\Delta x=0.02$.

tropy equation yields very satisfactory stable results with constant entropy, while the conservative form leads to a very rapid growth of entropy, due to spurious source terms arising from the lack of consistency with the LBM mass conservation. 


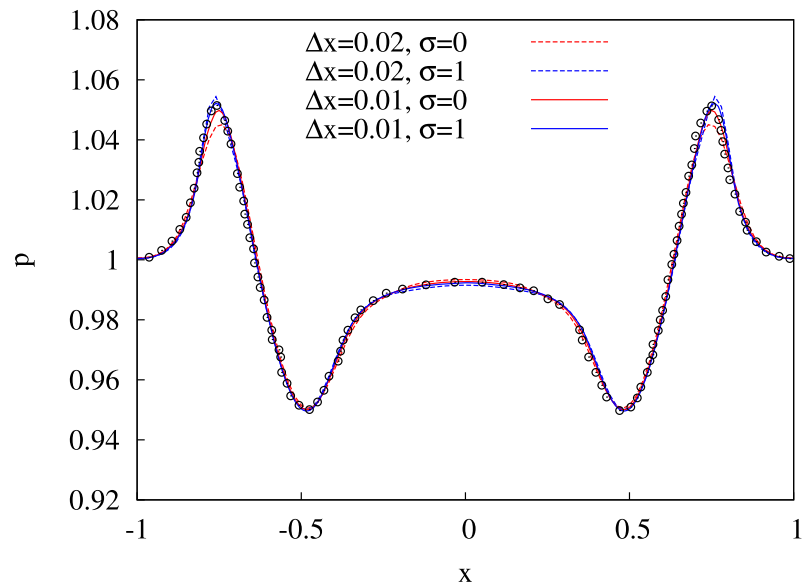

(a) pressure

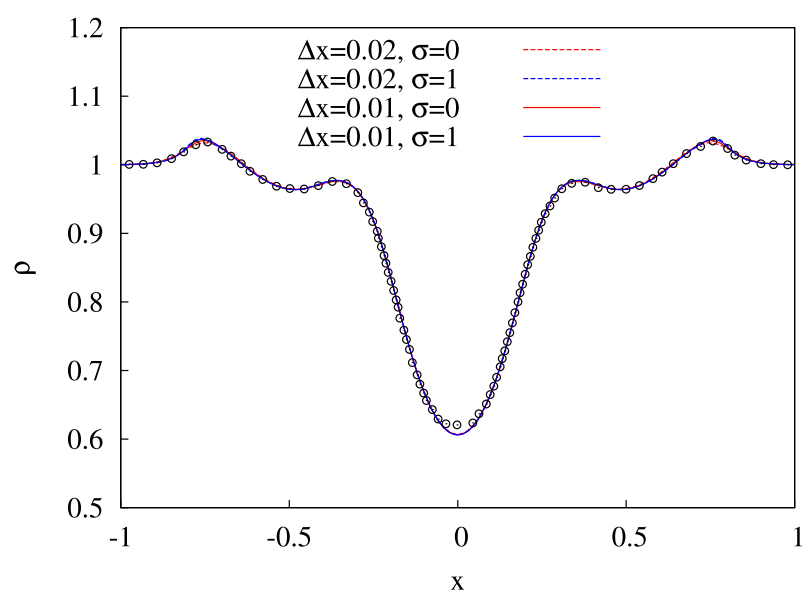

(c) density

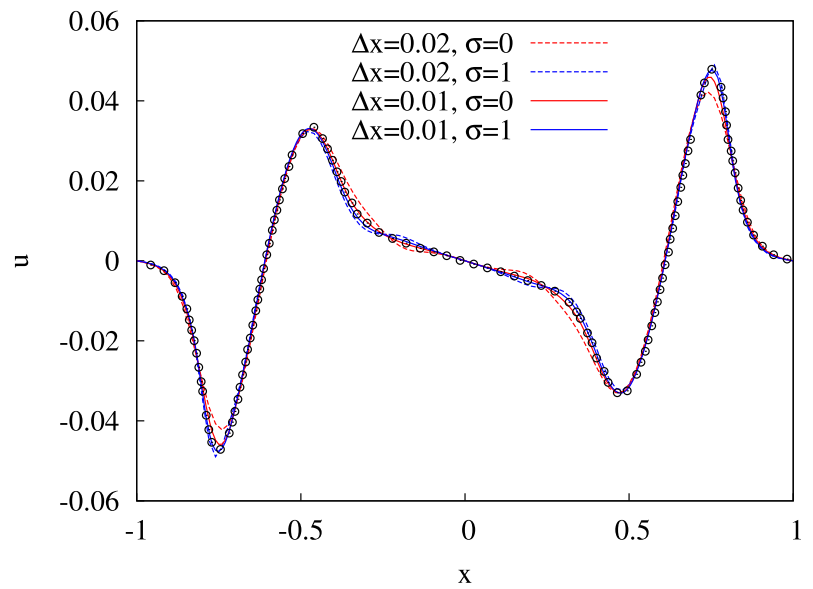

(b) velocity

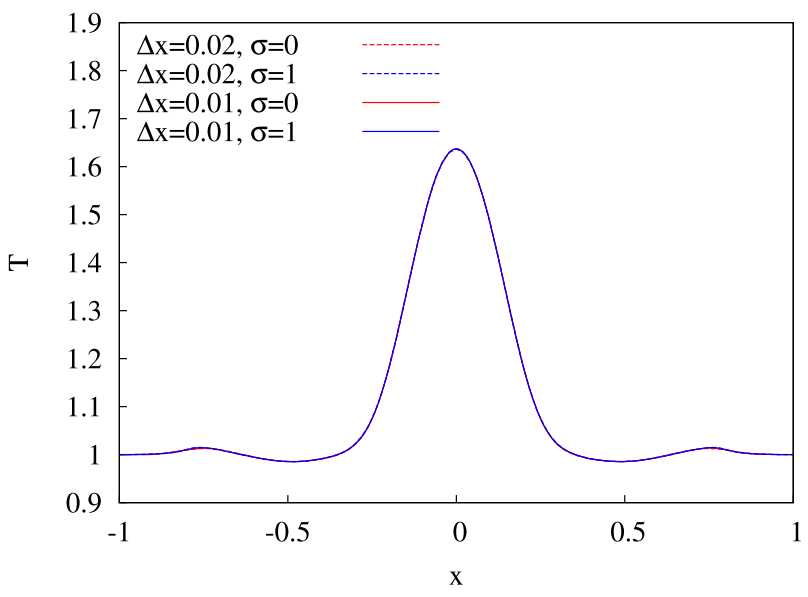

(d) temperature

Fig. 6. Comparison of profiles along $x$-direction middle line. (Lines: present LBM, symbols: reference solution obtained by grid spacing of $\Delta x=0.0002$ ).

\subsection{Non-isothermal Gaussian pulse}

Here, we consider a three-dimensional thermal acoustic wave traveling in radial direction including temperature evolution. The aim of this test case is to verify the preservation of isotropy by the present D3Q19 model and the coupling between velocity, pressure and temperature. The initial condition is given by:

$$
\rho=1.0, p=1+\exp \left(-k r^{2}\right), u=0, v=0, w=0
$$

where $r=\left[\left(x-x_{c}\right)^{2}+\left(y-y_{c}\right)^{2}+\left(z-z_{c}\right)^{2}\right]^{1 / 2}$ is the distance from the pulse center $\left(x_{c}, y_{c}, z_{c}\right)=(0,0,0)$. The perturbation parameter is taken equal to $k=40$. The computational domain size is $[-1,1] \times[-1,1] \times[-1,1]$, with periodic boundary conditions. The present simulations are performed on $100 \times 100 \times 100(\Delta x=0.02)$ and $200 \times 200 \times 200(\Delta x=0.01)$ grids with $\delta_{t}$ of 0.00667 and 0.00333 , respectively.

The pressure, u-velocity, density and temperature fields at time $t_{\text {end }}=1.0$ obtained using pure recursive-regularization collision model (parameter $\sigma=1$ ) on grid resolution of $\Delta x=0.02$ are presented in Fig. 5. It is found that the isotropic evolution is well preserved by the compressible D3Q19 LB model.

Fig. 6 displays the profiles of pressure, horizontal velocity, density and temperature obtained by the proposed LB model using HRR parameter $\sigma=0$ and 1 on grid resolution of $\Delta x=0.02$ and 0.01 at time $t_{\text {end }}=1.0$. A very good agreement with the reference solution is obtained, showing the capability of the present method to capture thermodynamic couplings and nonlinear wave propagation. The computation of the reference solution was performed using a second-order TVD finite volume scheme with the Osher type flux using $10^{4}$ cells in $1 \mathrm{D}[84,85]$. It is observed that the small value of parameter $\sigma$ induces a finite dissipating on coarse grid resolution. The hyper-viscous feature of the HRR collision model is consistent with the two-dimensional LB model [40]. 


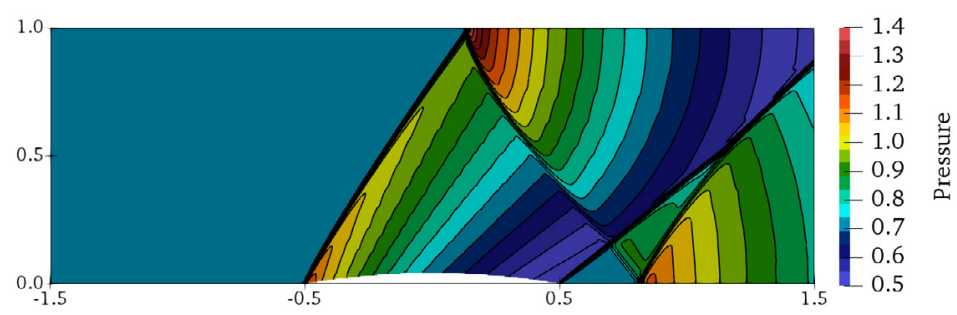

(a) pressure

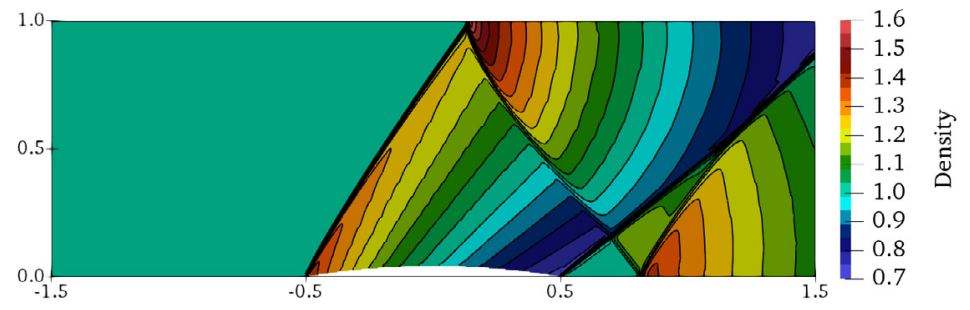

(b) density

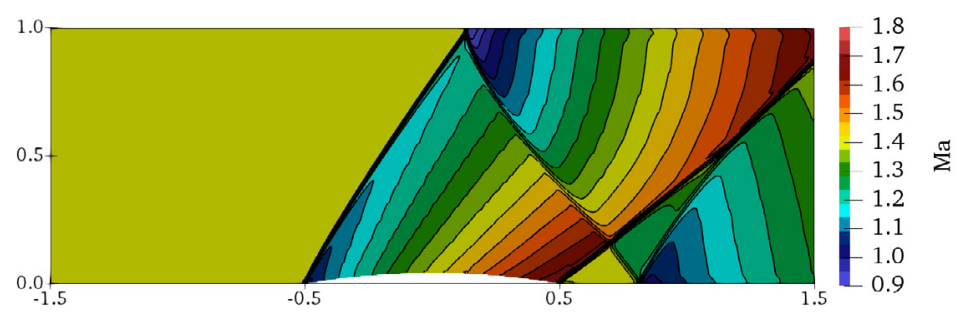

(c) Ma number

Fig. 7. Pressure, density and Mach number fields obtained by the present D3Q19 LB model on grid size $\Delta x=0.005$.

\subsection{Inviscid supersonic flow over a $4 \%$ circular bump}

The second case is a very classical one to investigate the accuracy and robustness of the numerical method when dealing with shock wave, i.e. the inviscid steady supersonic flow in a channel with a bump at inlet Mach number equal to 1.4 . The inviscid fluid behavior is mimicked in the present LBM by enforcing a very small molecular viscosity along with slip boundary conditions at solid wall (therefore preventing the growth of boundary layers). The domain of the channel is $x \in$ $[-1.5,1.5] \times y \in[0,1]$. The bump is set on the bottom of the channel from $x=-0.5$ to 0.5 . The height of the bump is 0.04 .

The inlet boundary of the domain is a supersonic inflow with the fixed values $\rho_{\infty}=1.0, p_{\infty}=1 / \gamma, T_{\infty}=p / \rho, u_{\infty}=$ 1.4. The supersonic outflow conditions are implemented on the opposite boundary. Other boundaries are free-slip adiabatic walls. In the simulations, the present LB model is assessed on $600 \times 200 \times 1(\Delta x=0.005)$ and $300 \times 100 \times 1(\Delta x=0.01)$ grids with the time step $\delta_{t}$ being 0.00166 and 0.00333 , respectively. The value of HRR parameter $\sigma$ is set to 0.9 .

Fig. 7 shows the pressure, density and Mach number fields obtained on the fine grid. It can be seen that the reflection and interaction of the shock waves are very well captured without nonphysical wiggles. As a quantitative comparison, the Mach number distributions on the bottom and top walls are plotted in Fig. 8. The reference results marked by the symbols were reported in Ref. [86]. The reference solution was obtained using the artificial compression method (ACM) with a second-order accuracy on $90 \times 30$ nonuniform body-fitted grids. In the comparison, it can be found that both the results on coarse and fine grids are in excellent agreement with the reference results.

The pressure and Mach number profiles along horizontal $(y=0.5)$ and vertical $(x=0)$ mid-line are shown in Fig. 9. The reference results are obtained using the Roe scheme and JST scheme implemented in a second-order accurate finite volume solver (FVM) for the Euler equations. Both the solutions of the LBM and the FVM are computed on the uniform grid spacing $\Delta x=0.005$. From this figure, it can be seen that the results obtained by the present LBM are very close to those by the FVM.

\subsection{Spherical explosion in a 3D enclosed box}

A spherical explosion in a 3D enclosed box is considered to assess capability of the present model to capture three dimensional complex shock waves. The numerical test is an unsteady compressible flow. The initial condition of this problem are illustrated in Fig. 10. 


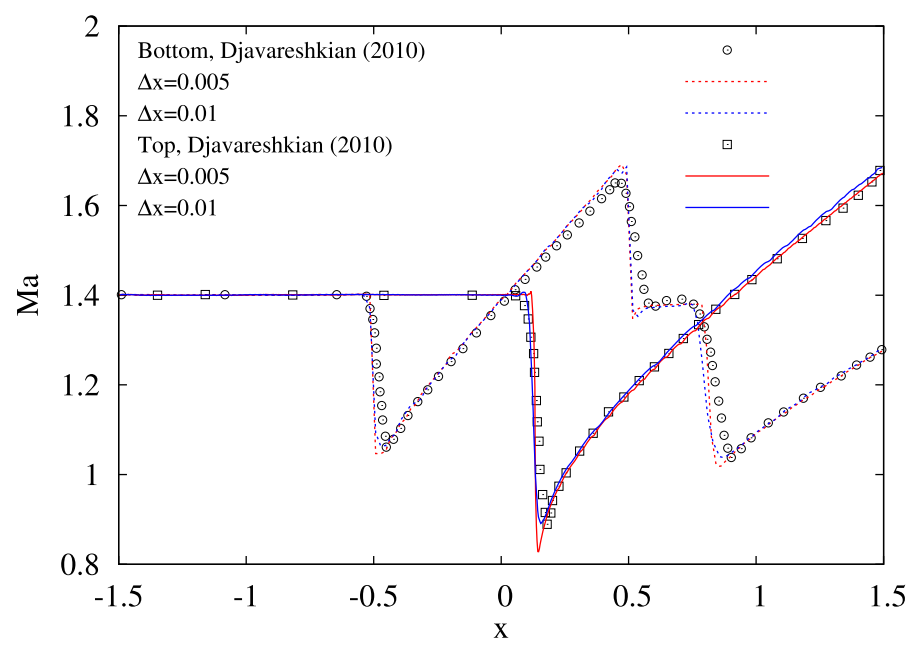

Fig. 8. Mach number distributions obtained by the present D3Q19 LB model. (Lines: present results, symbols: reference solution in Ref. [86].)

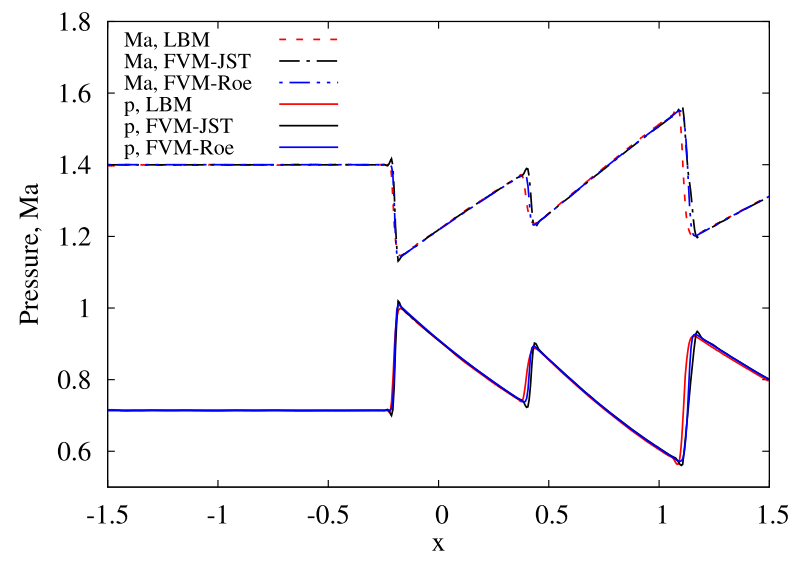

(a) horizontal mid-line

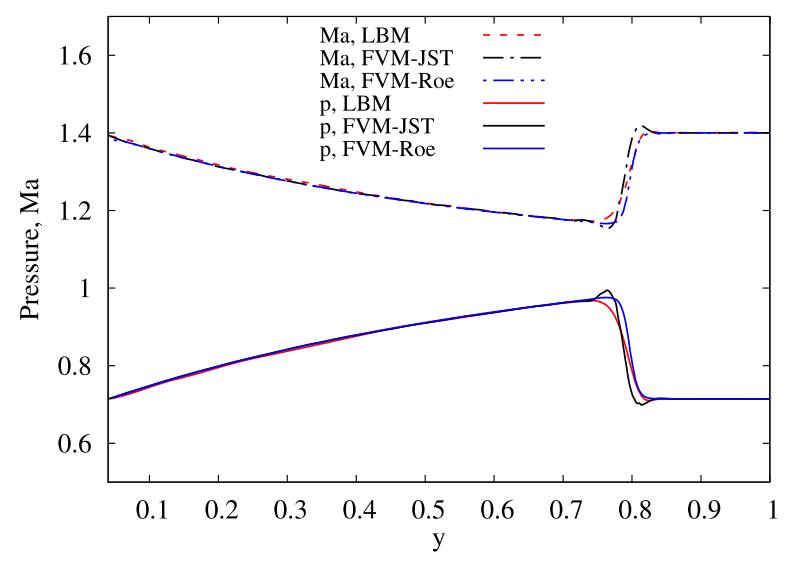

(b) vertical mid-line

Fig. 9. Mach number and pressure distributions along mid-line. The reference solution is computed using a classical Euler solver based on the Finite Volume Method with the second-order Roe scheme and JST scheme.

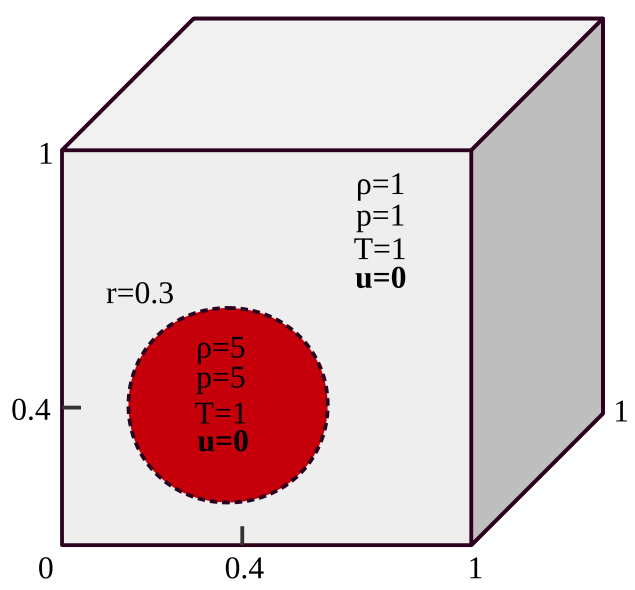

Fig. 10. Initial condition for spherical explosion in 3D enclosed box. 


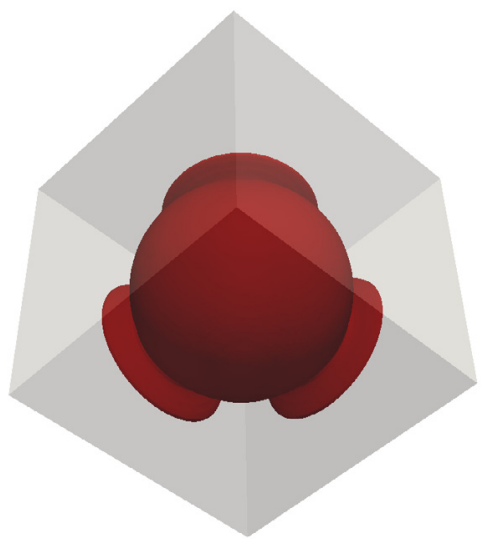

(a) $\mathrm{t}=0.125$

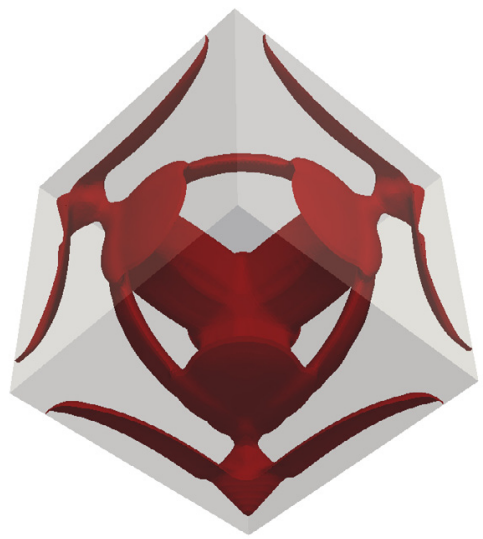

(c) $\mathrm{t}=0.375$

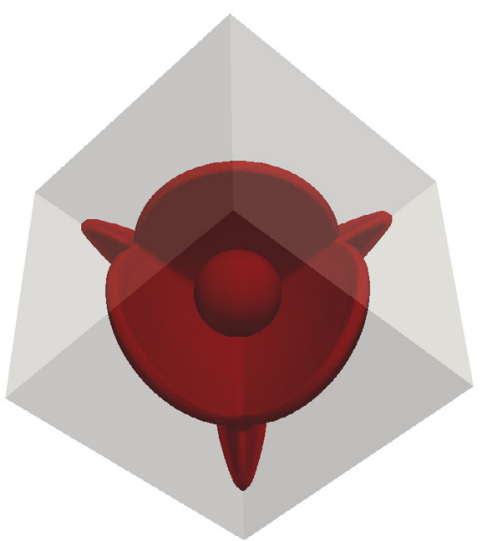

(b) $\mathrm{t}=0.25$

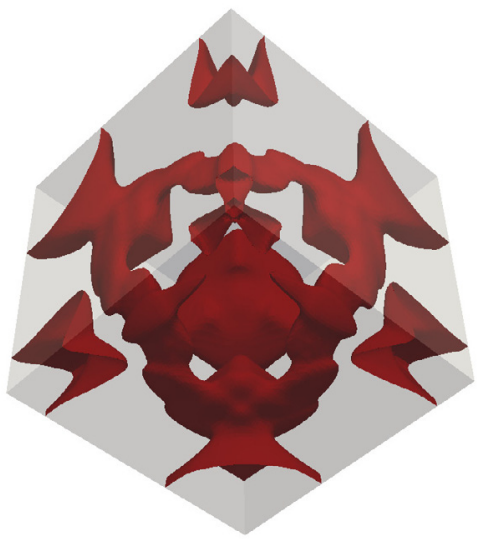

(d) $\mathrm{t}=0.5$

Fig. 11. Instantaneous density isosurface of $\rho=1.8$ in the 3D enclosed box.

The computational domain is a unit cube and all the boundary conditions are free-slip adiabatic walls. The computational grid resolution is $100 \times 100 \times 100$ and time step taken equal to $\delta_{t}=0.00333$. The HRR parameter $\sigma$ is set to 0.7 .

The density iso-surfaces $\rho=1.8$ obtained at $t=0.125,0.25,0.375$ and 0.5 are shown in Fig. 11. The instantaneous evolution agrees well with the reference solution presented in [87].

In order to further compare our results with other solutions, the density contours in the $z=0.4$ plane at $t=0.5$ obtained by present LB model and by the finite volume solver are presented in Fig. 12. It can be found that the complex flow features such as the shock wave interactions are well captured by the present model. The density contours computed via LBM are similar with those of the FVM solution. For the quantitative comparison, the density, temperature and Ma number profiles obtained by the above two methods at $t=0.5$ are shown in Fig. 13. It is seen that the present LB results are in good agreement with the results of FVM.

\subsection{Shock-vortex interactions}

This test case deals with the interaction of a stationary normal shock wave with a single vortex. The shock wave is defined by an upstream Mach number $M_{s}$. The right and left states of the normal shock are as follows:

\begin{tabular}{ll}
\hline$\rho_{R}=1.0$ & $\rho_{L}=\frac{(\gamma+1) M_{S}^{2}}{2+(\gamma-1) M_{S}^{2}} \rho_{R}$ \\
$p_{R}=1.0$ & $p_{L}=\left(\frac{2 \gamma}{\gamma+1} M_{s}^{2}-\frac{\gamma-1}{\gamma+1}\right) p_{R}$ \\
$T_{R}=p_{R} / \rho_{R}$ & $T_{L}=p_{L} / \rho_{L}$ \\
$u_{R}=-M_{S}$ & $u_{L}=-\frac{2+(\gamma-1) M_{S}^{2}}{(\gamma+1) M_{S}^{2}} M_{S}$ \\
$v_{R}=0$ & $v_{L}=0$ \\
\hline
\end{tabular}




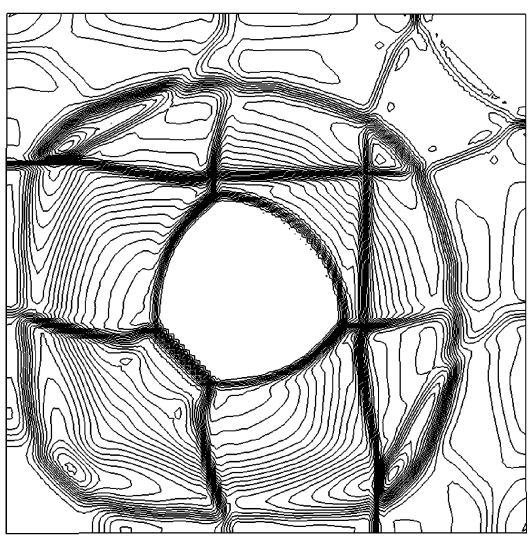

(a) LBM solution

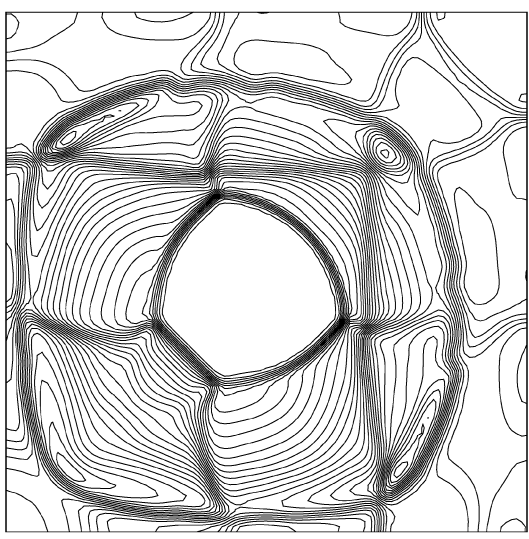

(b) FVM-Roe solution

Fig. 12. Density contours in a $2 \mathrm{D}$ slice through $z=0.4$ at $t=0.5$. (a) the present solution obtained by HRR-LB model on $100 \times 100 \times 100$ grids, $(\mathrm{b})$ the reference solution computed by a finite volume solver with the second-order Roe scheme on the same grid resolution. The contours are displayed from 0.1 to 2.6 with 40 levels in the both figures.

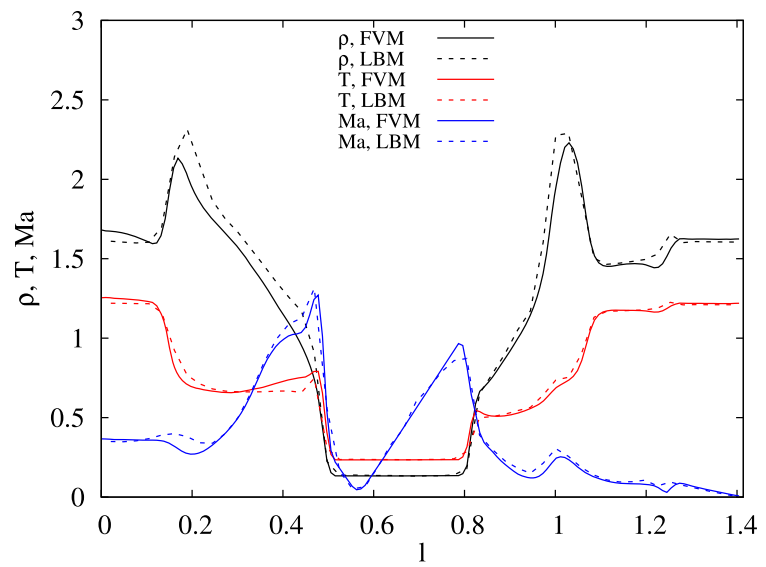

(a) $45^{\circ}$ line

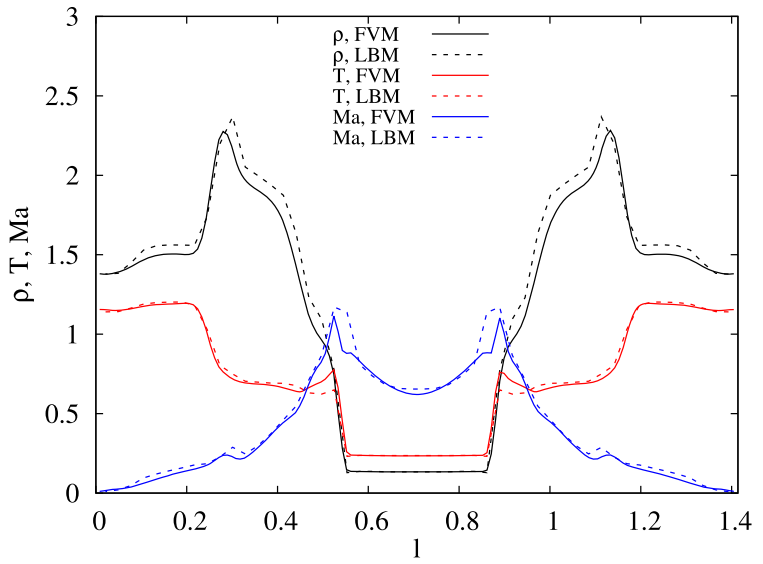

(b) $135^{\circ}$ line

Fig. 13. Density, temperature and Ma number profile along cross lines in a 2D slice through $z=0.4$ at $t=0.5$. The present solution is obtained by HRR-LB model on $100 \times 100 \times 100$ grids, and the reference solution computed by a finite volume solver with the second-order Roe scheme on the same grid resolution.

The initial density, pressure, tangential and radial velocities of the vortex are expressed by

$$
\begin{aligned}
& \rho_{\theta}(r)=\left[1-\frac{\gamma-1}{2} M_{v}^{2} r \exp \left(1-r^{2}\right)\right]^{\frac{1}{\gamma-1}}, p(r)=\frac{1}{\gamma} \rho^{\gamma}(r) . \\
& u_{\theta}(r)=M_{v} r \exp \left[\left(1-r^{2}\right) / 2\right], u_{r}(r)=0,
\end{aligned}
$$

where the distance from the vortex core $r$ is non-dimensionalized by the vortex radius $R$.

The above flow field of vortex is added to the upstream of the shock wave at initial time. The following flow parameters are used in the test:

$$
M_{S}=1.2, M_{v}=0.25, \operatorname{Re}=800, R=1, \gamma=1.4
$$

The Reynolds number is defined by $R e=\rho_{R} a_{R} R / \mu$ with $a_{R}$ being the sound speed of the upstream of the shock. A computational domain $[-20 \mathrm{R}, 8 \mathrm{R}] \times[-12 \mathrm{R}, 12 \mathrm{R}]$ is considered in the simulation. Initially the single vortex is located at $x=2 R$ and $y=0$, and the planar shock wave is specified at $x=0$ by imposing density, velocity and pressure variables corresponding to the above left and right states of the normal shock.

In this simulation, the grid resolution is $1120 \times 960 \times 1$, the time step is set to $\delta_{t}=0.00833$ and the HRR parameter $\sigma$ is taken equal to 0.7 . The pressure fields at different time are shown in Fig. 14 . Here, the sound pressure is $\Delta p=\left(p-p_{L}\right) / p_{L}$. The sound pressure contours are from -0.48 to 0.16 with 60 levels. It can be found that these results are very similar with the sound pressure fields given in Ref. [88]. 


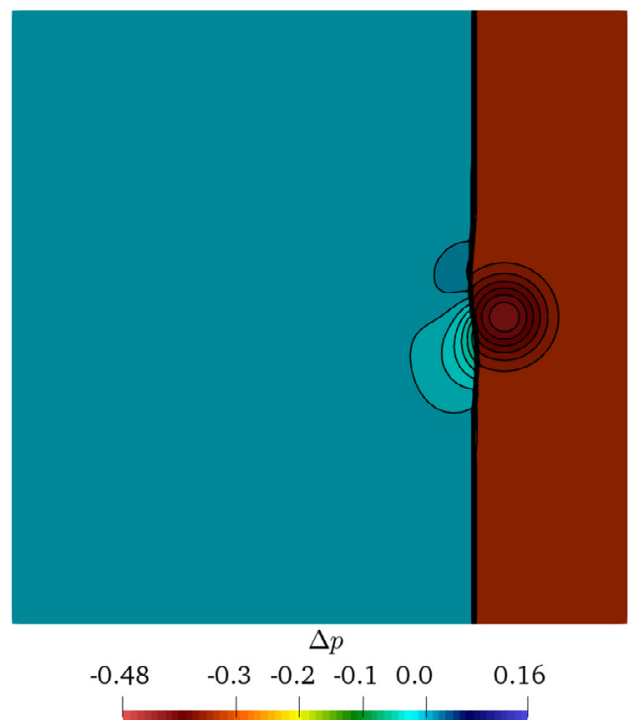

(a) $\mathrm{t}=1 \mathrm{~T}$

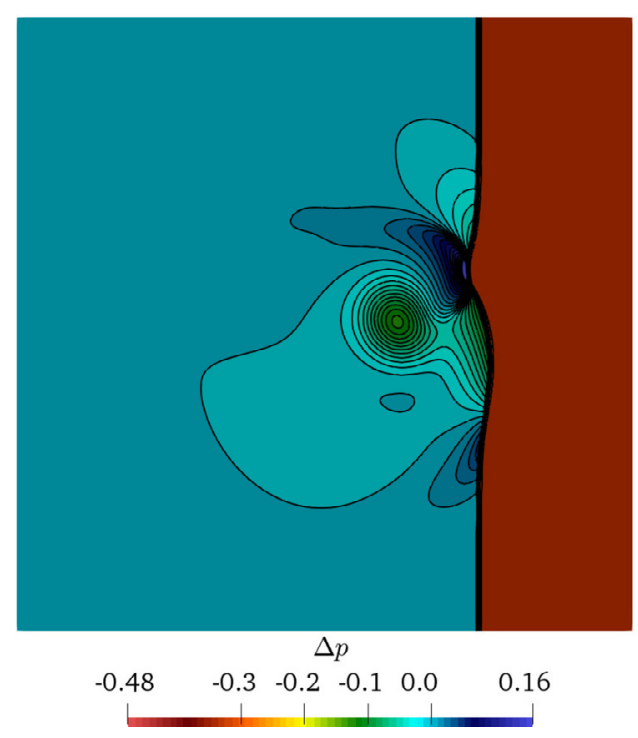

(c) $\mathrm{t}=4 \mathrm{~T}$

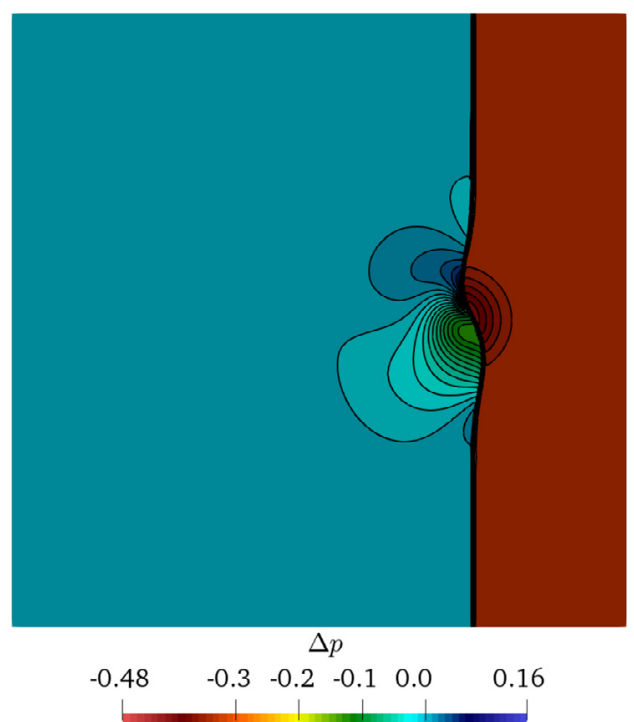

(b) $\mathrm{t}=2 \mathrm{~T}$

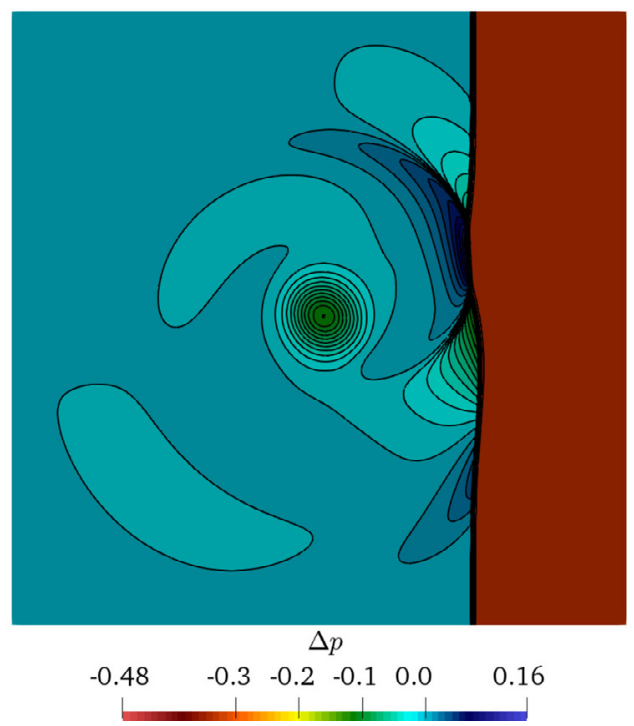

(d) $\mathrm{t}=6 \mathrm{~T}$

Fig. 14. Instantaneous sound pressure contours obtained by HRR-LB model on $1120 \times 960 \times 1$ grids. The sound pressure $\Delta p=\left(p-p_{L}\right) / p_{L}$ contours are from -0.48 to 0.16 with 60 levels.

The radial distributions of the sound pressure are plotted in Fig. 15. In the figure, $r$ is the distance from the center of the vortex with a fixed angle $\theta=-45^{\circ}$. Fig. 16 is the circumferential distributions of the sound pressure at $t=6 T$. In those two figures, the results represented by symbols are from Ref. [88]. Those reference results were obtained using a finite difference method with a sixth-order-accurate compact scheme in space and the fourth-order Runge-Kutta scheme for time-integration on $1044 \times 1170$ non-uniform grids. It can be observed that the results obtained by LB are in very good agreement with the reference results.

\subsection{Compressible laminar flow over flat plate}

The compressible laminar flow over flat plate has been investigated numerically and theoretically over the years [89,90]. Here, we use this problem to assess the capability of the present method on handling the viscous effects. Considering a compressible flow with upstream Mach number $M a_{\infty}=0.5,1.0,1.5$ over a plate of length $L$, the computational domain is $x \in[-0.25,1] \times y \in[0, h] \times z \in[0, \Delta x]$. Here, $\Delta x$ denotes the mesh size which is $\Delta x=1.25 \times 10^{-3}$. $h$ is set to 1.25 


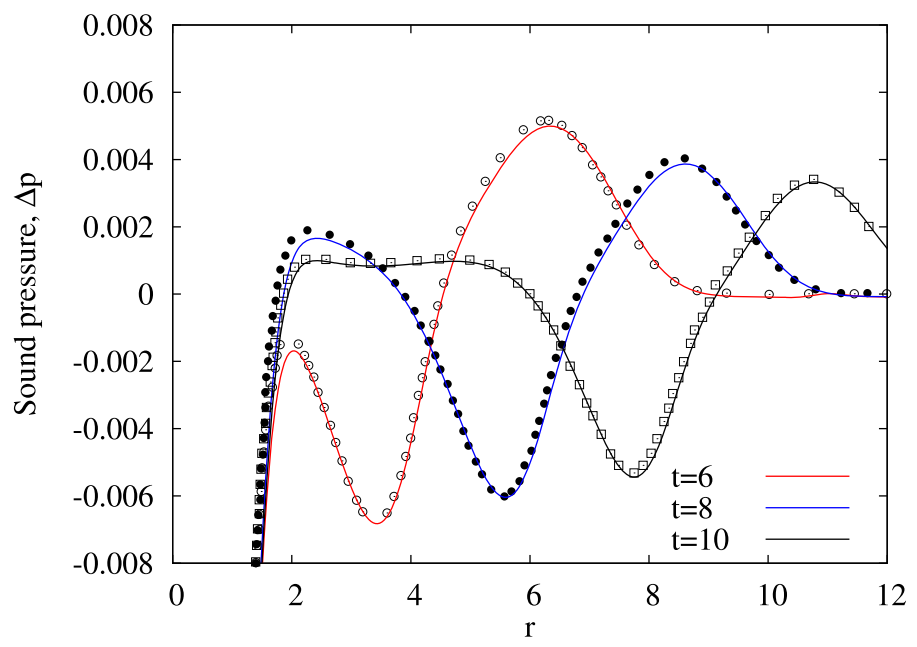

Fig. 15. Radial distributions of the sound pressure $\Delta p . r$ is the distance from the center of the vortex with a fixed angle $\theta=-45^{\circ}$. The solid lines represent the present LB solution and the symbols denote the reference solution in Ref. [88].

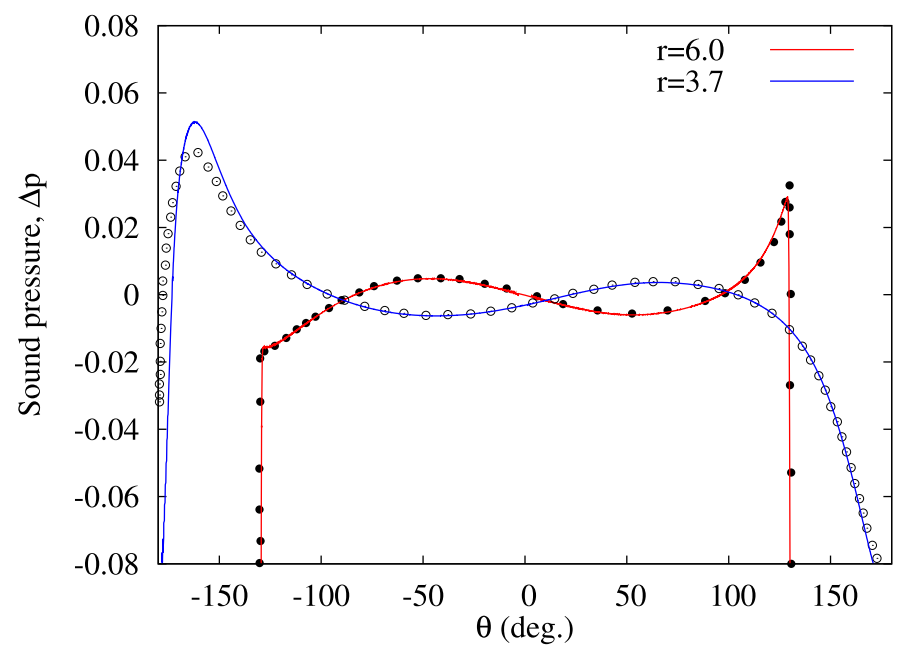

Fig. 16. Circumferential distributions of the sound pressure $\Delta p$ at $t=6 T$. The solid lines represent the present LB solution and the symbols denote the reference solution in Ref. [88].

for Mach number 1.5 and 1.0. For Mach 0.5, one takes $h=0.25$. The HRR parameter is set to $\sigma=0.9$ and the time step $\delta_{t}=4.166 \times 10^{-4}$. The boundary conditions are set as follows:

- Symmetry BC on at $y=0,-0.25 \leq x<0$.

- No-slip, adiabatic BC on $y=0,0 \leq x \leq 1$.

- Inflow BC at $x=0$ with $\rho_{i n}=1$, and $(u, v)_{\text {in }}=\left(u_{\infty}, 0\right)$.

- Subsonic outflow at $y=h$ and $x=1$ with pressure $p_{\text {out }}=1 / \gamma$ for $M a_{\infty}=0.5$.

- Supersonic outflow at $y=h$ and $x=1$ for $M a_{\infty}=1.0,1.5$.

In order to obtain a steady laminar solution, the Reynolds number is taken as $10^{4}$. A variable temperature-dependent viscosity given by $\rho \mu=\rho_{\infty} \mu_{\infty}$ is used in this simulation. The Prandtl number is taken equal to 1.0. These viscosity and $\mathrm{Pr}$ are set according to the reference similarity solutions of compressible boundary layer [91].

Fig. 17 displays the contours of Mach number obtained by the present LB model at Ma=1.5. Fig. 18 shows the profiles of horizontal velocity, temperature, density and skin friction obtained by the present LB model. In the figure, $\eta$ is the dimensionless coordinate with Illingworth transform which is defined as the one in reference [40]. The reference solution of temperature and density are obtained using the way in [40]. $C_{w}$ is the Chapman-Rubesin parameter which is equal to 1 under the condition of $\rho \mu=\rho_{\infty} \mu_{\infty}$. From the figure, it can be found that the simulated velocity profiles and reference solution are in a very good agreement at $M a_{\infty}=0.5,1.0,1.5$. This kind of good agreement can also be found between the skin friction predicted by the LB method and the ones of the Blasius solution. For the density and temperature, the results 


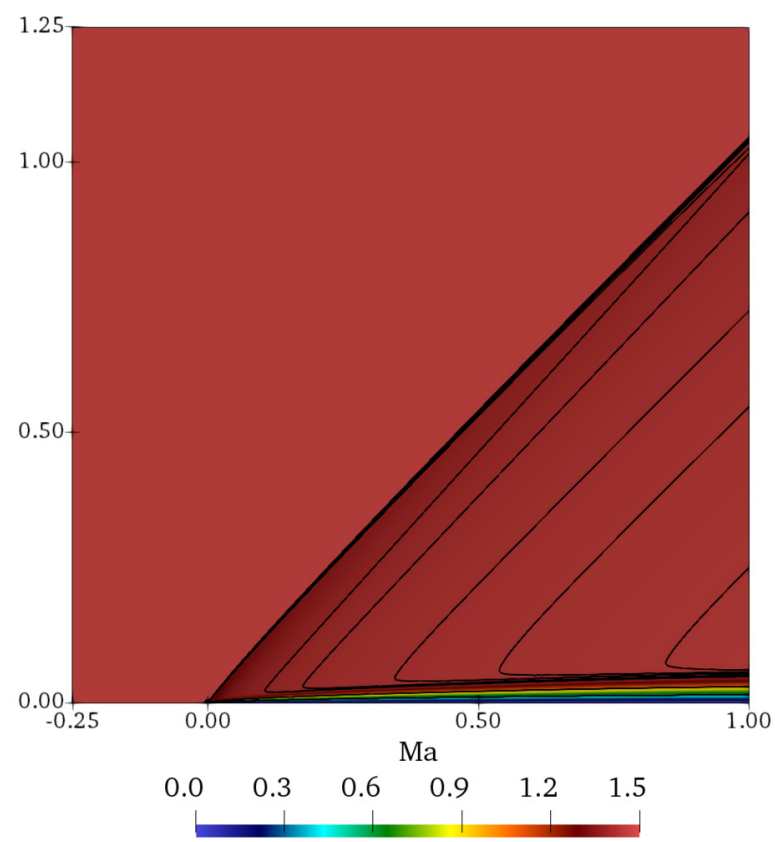

Fig. 17. Mach number field of laminar boundary layer problem at $M a_{\infty}=1.5$.
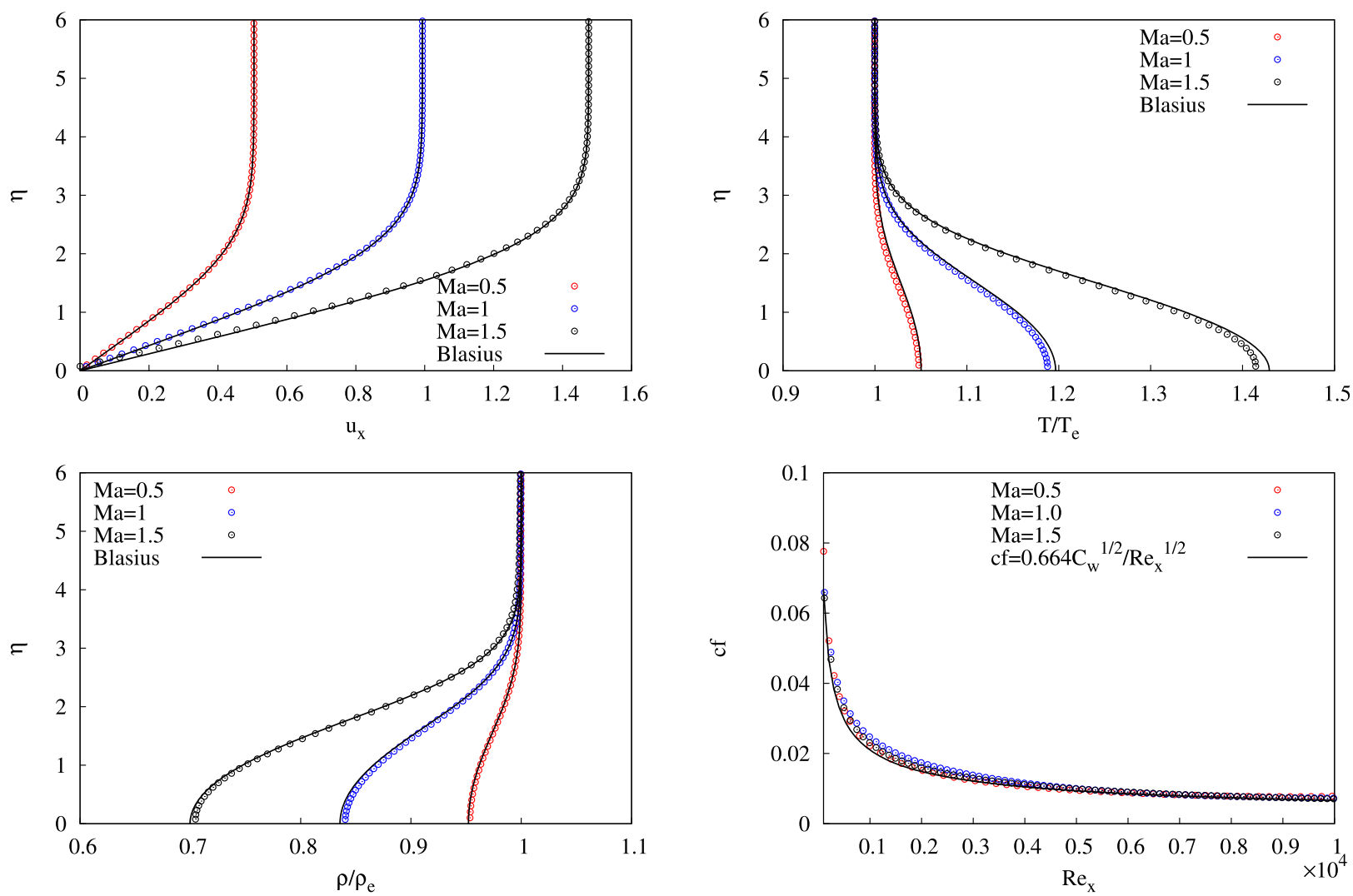

Fig. 18. Profile of horizontal velocity, temperature, density and skin friction obtained by the LB method on laminar flow over flat plate at $M a_{\infty}=0.5,1.0$ and 1.5. The solid lines represent Blasius solution at the corresponding Ma number. 
obtained by the present LB method and the reference solution are also matched very well at $M a_{\infty}=0.5,1.0,1.5$. Thus, the present LB model can simulate the problem of coupling between fluid flow and heat transfer with high accuracy.

\section{Conclusions}

In order to efficiently simulate the subsonic to supersonic compressible 3D flows with shocks, an extended hybrid lattice Boltzmann method is proposed. In this method, the mass and momentum conservation equations are solved using an extended lattice Boltzmann model with a hybrid recursive regularization collision operator on D3Q19 lattice stencil. Optimized form of the collision kernel, Hermite basis and the correction terms have been proposed. The energy conservation law is implemented by solving the entropy conservation equation written in non-conservative form to minimize the influence of high-order errors on mass conservation stemming from LBM on a finite volume framework. The total number of degrees of freedom per cell is 20, which is among the most efficient existing LB methods for high speed compressible flows. The isotropic feature is well preserved by the revised equilibrium distribution function on D3Q19 lattice. Numerical tests show that this extended hybrid method can accurately and efficiently simulate the compressible flow problems with shock waves including the shock capturing, the reflection and interaction of shock waves, shock-vortex interaction and the problem of supersonic laminar boundary layer. The proposed method is well suited for supersonic flows without strong non-equilibrium effects, for which the imposed Q19 lattice would not be accurate enough. To handle rarefied gas dynamics, hypersonic flows or reactive plasma flows higher order lattices of non-LB methods with optimized selection of discrete velocities should certainly be considered.

The theoretical demonstration of the convergence of the present method toward the Navier-Stokes solutions in the presence of shock waves remains an open issue. The answer to this question is quite difficult. In the view of conventional fluid dynamics, a non-conservative formulation of the hyperbolic system and getting the correct solution has been a longstanding debate [92-95]. In the view of the kinetic theory, the Navier-Stokes equations derived from the Boltzmann equation are not hyperbolic, and do not allow discontinuous shocks, where the use of artificial viscosity can be considered as a regularization of Chapman-Enskog expansion [96,97]. As a computational proof, the test cases in this paper empirically demonstrate the capability of the hybrid LB method for compressible flows with shock waves. Further theoretical study will be devoted to this special question in the future work.

\section{Declaration of competing interest}

The authors declare that they have no known competing financial interests or personal relationships that could have appeared to influence the work reported in this paper.

\section{Acknowledgements}

This work was granted access to the HPC resources of Aix-Marseille Université financed by the project Equip@Meso (ANR10-EQPX-29-01) of the program "Investissements d'Avenir" supervised by the Agence Nationale de la Recherche. This work was performed using HPC resources from GENCI-TGCC/CINES (Grant 2018-A0052A07679). This work was also supported by project of ProLB software (http://www.prolb-cfd.com). Part of this research was supported by ANR, Renault, Airbus and SafranTech by the Industrial Chair Program ALBUMS (ANR-CHIND-18-ALBUMS).

\section{Appendix A. Chapman-Enskog analysis}

In order to derive macroscopic equations, analyze the numerical stability issue induced by truncation error of correction terms and construct the stable discretization for the correction terms, the Chapman-Enskog analysis for the present method is present in the appendix. First, the density distribution function $f_{i}$ is expanded around the $f_{i}^{e q}$ distributions as follows:

$$
f_{i}=f_{i}^{(0)}+\epsilon f_{i}^{(1)}+\epsilon^{2} f_{i}^{(2)}+\cdots
$$

with

$$
\sum_{i} f_{i}^{(n)}=0, \quad \sum_{i} c_{i \alpha} f_{i}^{(n)}=0, n>0
$$

The correction term $\psi_{i}$ can be expanded as $\psi_{i}=\epsilon \psi_{i}^{(0)}$. By matching the scales of $\epsilon^{1}, \epsilon^{2}$ we have

$$
\begin{aligned}
& \epsilon^{1}:\left(\frac{\partial}{\partial t_{1}}+c_{i \alpha} \frac{\partial}{\partial x_{1 \alpha}}\right) f_{i}^{e q}+\frac{f_{i}^{(1)}}{\tau}=\psi_{i}^{(0)} \\
& \epsilon^{2}: \frac{\partial f_{i}^{e q}}{\partial t_{2}}+\left(\frac{\partial}{\partial t_{1}}+c_{i \alpha} \frac{\partial}{\partial x_{1 \alpha}}\right) f_{i}^{(1)}+\frac{f_{i}^{(2)}}{\tau}=0
\end{aligned}
$$


Considering that the correcting term $\psi_{i}$ is approximated in the second-order finite difference scheme, it can be given as

$$
\sum_{i} c_{i \alpha} c_{i \beta} \psi_{i}=-\frac{\partial}{\partial x_{\gamma}} \Psi_{\alpha \beta \gamma}-\lambda_{3}(\Delta x)^{2} \frac{\partial^{3}}{\partial x_{\gamma}^{3}} \Psi_{\alpha \beta \gamma}-\lambda_{4}(\Delta x)^{3} \frac{\partial^{4}}{\partial x_{\gamma}^{4}} \Psi_{\alpha \beta \gamma}-\mathcal{O}\left(\Delta x^{4}\right)
$$

where $\lambda_{3}, \lambda_{4}$ are respectively coefficient of the third and forth order truncation errors. $\Psi_{\alpha \beta \gamma}$ is the deviation term of the third-order moment between the exact Maxwellian distribution and the discrete equilibrium distribution function, which has been defined in Eq. (4) and summarized in Table 3. Note that only one of $\gamma$ is dummy index in the spatial coordinates of the high order derivatives for $\Psi_{\alpha \beta \gamma}$.

The $t_{1}$ order of the continuity equation, momentum equation and energy equation can be derived as

$$
\begin{aligned}
& \frac{\partial \rho}{\partial t_{1}}+\frac{\partial}{\partial x_{1 \alpha}}\left(\rho u_{\alpha}\right)=0 \\
& \frac{\partial}{\partial t_{1}}\left(\rho u_{\alpha}\right)+\frac{\partial}{\partial x_{1 \beta}}\left(\rho u_{\alpha} u_{\beta}+p \delta_{\alpha \beta}\right)=0 \\
& \frac{\partial}{\partial t_{1}}\left(\rho E_{m}\right)+\frac{\partial}{\partial x_{1 \beta}}\left[\left(\rho E_{m}+p\right) u_{\beta}\right]=\lambda_{3}(\Delta x)^{2} \frac{\partial^{3}}{\partial x_{\gamma}^{3}}\left[\left(1-\theta-u_{\gamma}^{2}\right) \rho u_{\gamma}\right]+\lambda_{4}(\Delta x)^{3} \frac{\partial^{4}}{\partial x_{\gamma}^{4}}\left[\left(1-\theta-u_{\gamma}^{2}\right) \rho u_{\gamma}\right]
\end{aligned}
$$

where $E_{m}=\left(u^{2}+3 c_{s}^{2} \theta\right) / 2$ is total energy of monoatomic gas in the D3Q19 model. A pressure equation can be derived from the above energy equation as follows

$$
\frac{\partial p}{\partial t_{1}}+\frac{\partial}{\partial x_{1 \gamma}}\left(p u_{\gamma}\right)+\frac{2}{3} p \frac{\partial u_{\gamma}}{\partial x_{1 \gamma}}=0
$$

The $t_{2}$ order of the continuity equation and momentum equation can be derived as

$$
\begin{aligned}
& \frac{\partial \rho}{\partial t_{2}}=0 \\
& \frac{\partial}{\partial t_{2}}\left(\rho u_{\alpha}\right)+\frac{\partial}{\partial x_{1 \beta}}\left(\sum_{i} c_{i \alpha} c_{i \beta} f_{i}^{(1)}\right)=0
\end{aligned}
$$

After some algebra, one obtains

$$
\begin{aligned}
\sum_{i} c_{i \alpha} c_{i \beta} f_{i}^{(1)} & =-\tau \sum_{i} c_{i \alpha} c_{i \beta}\left[\left(\frac{\partial}{\partial t_{1}}+c_{i \gamma} \frac{\partial}{\partial x_{1 \gamma}}\right) f_{i}^{e q}-\psi_{i}^{(0)}\right] \\
& =-\tau\left[\frac{\partial}{\partial t_{1}}\left(\rho u_{\alpha} u_{\beta}+p \delta_{\alpha \beta}\right)+\frac{\partial}{\partial x_{\gamma}} \Psi_{\alpha \beta \gamma}+\frac{\partial}{\partial x_{1 \gamma}}\left(p[u \delta]_{\alpha \beta \gamma}+\rho u_{\alpha} u_{\beta} u_{\gamma}-\Psi_{\alpha \beta \gamma}\right)\right] \\
& =-\tau p\left[\frac{\partial u_{\beta}}{\partial x_{1 \alpha}}+\frac{\partial u_{\alpha}}{\partial x_{1 \beta}}-\frac{2}{3} \frac{\partial u_{\gamma}}{\partial x_{1 \gamma}} \delta_{\alpha \beta}\right]-\tau\left[\lambda_{3}(\Delta x)^{2} \frac{\partial^{3}}{\partial x_{\gamma}^{3}} \Psi_{\alpha \beta \gamma}+\lambda_{4}(\Delta x)^{3} \frac{\partial^{4}}{\partial x_{\gamma}^{4}} \Psi_{\alpha \beta \gamma}\right]
\end{aligned}
$$

The following equations with the truncation errors can be finally obtained:

$$
\begin{aligned}
\frac{\partial \rho}{\partial t}+\frac{\partial}{\partial x_{\alpha}}\left(\rho u_{\alpha}\right)=0 & \\
\frac{\partial}{\partial t}\left(\rho u_{\alpha}\right)+\frac{\partial}{\partial x_{\beta}}\left(\rho u_{\alpha} u_{\beta}+p \delta_{\alpha \beta}\right) & =\frac{\partial}{\partial x_{\beta}}\left[\mu\left(\frac{\partial u_{\beta}}{\partial x_{\alpha}}+\frac{\partial u_{\alpha}}{\partial x_{\beta}}-\frac{2}{3} \frac{\partial u_{\gamma}}{\partial x_{\gamma}} \delta_{\alpha \beta}\right)\right] \\
& +\frac{\partial}{\partial x_{\beta}}\left\{\tau\left[\lambda_{3}(\Delta x)^{2} \frac{\partial^{3}}{\partial x_{\gamma}^{3}} \Psi_{\alpha \beta \gamma}+\lambda_{4}(\Delta x)^{3} \frac{\partial^{4}}{\partial x_{\gamma}^{4}} \Psi_{\alpha \beta \gamma}\right]\right\} \\
\frac{\partial}{\partial t_{1}}\left(\rho E_{m}\right)+\frac{\partial}{\partial x_{1 \beta}}\left[\left(\rho E_{m}+p\right) u_{\beta}\right] & =\lambda_{3}(\Delta x)^{2} \frac{\partial^{3}}{\partial x_{\gamma}^{3}}\left[\left(1-\theta-u_{\gamma}^{2}\right) \rho u_{\gamma}\right]+\lambda_{4}(\Delta x)^{3} \frac{\partial^{4}}{\partial x_{\gamma}^{4}}\left[\left(1-\theta-u_{\gamma}^{2}\right) \rho u_{\gamma}\right]
\end{aligned}
$$

For simplify the analysis of stabilizing effect of the truncation error (TE), the one-dimensional dissipating term can be approximately given as follows:

In right-hand side of the momentum equation, 


$$
\begin{aligned}
\mathrm{TE}_{\mathrm{m}} & \approx \tau \lambda_{3}(\Delta x)^{2} \frac{\partial^{4}}{\partial x^{4}}\left[\left(\theta-1+u^{2}\right) \rho u\right] \\
& =\tau \lambda_{3}(\Delta x)^{2} \frac{\partial^{4}}{\partial x^{4}}\left[\rho u\left(2 E_{m}-1\right)\right] \\
& =2 \tau \lambda_{3}(\Delta x)^{2} \frac{\partial^{4}}{\partial x^{4}}\left(\rho u E_{m}\right)-\tau \lambda_{3}(\Delta x)^{2} \frac{\partial^{4}}{\partial x^{4}}(\rho u)
\end{aligned}
$$

In the RHS of the convective scale energy equation,

$$
\begin{aligned}
\mathrm{TE}_{\mathrm{e}} & \approx-\lambda_{4}(\Delta x)^{3} \frac{\partial^{4}}{\partial x^{4}}\left[\left(\theta-1+u^{2}\right) \rho u\right] \\
& =-\lambda_{4}(\Delta x)^{3} \frac{\partial^{4}}{\partial x^{4}}\left[\rho u\left(2 E_{m}-1\right)\right] \\
& =-2 \lambda_{4}(\Delta x)^{3} \frac{\partial^{4}}{\partial x^{4}}\left(\rho u E_{m}\right)+\lambda_{4}(\Delta x)^{3} \frac{\partial^{4}}{\partial x^{4}}(\rho u)
\end{aligned}
$$

It can be seen that the truncation error plays a role of dissipation on the momentum as well as thermal and kinetic energy fluctuations. $\lambda_{3}, \lambda_{4}$ are respectively Taylor's coefficient of the third and forth order truncation errors for the finite difference schemes, e.g., $\lambda_{3}=1 / 6, \lambda_{4}=0$ in the second-order central difference scheme and $\lambda_{3}=-1 / 3, \lambda_{4}=1 / 2$ in the second-order upwind difference scheme.

Compared to the negative coefficient of the forth-order term in Jameson's scheme [73], the negative value of both $\lambda_{3}$ and $-\lambda_{4}$ are required to maintain effective dissipation for high Mach compressible flows. Therefore, a half central difference and a half second-order upwind difference is used as a low-dissipative second-order upwind scheme with $\lambda_{3}=-1 / 12$ and $\lambda_{4}=1 / 4$ is adopted in this study, i.e., for $u>0$

$$
\frac{\partial \Psi}{\partial x}=\frac{1}{4 \Delta x}\left(\Psi_{i-2}-5 \Psi_{i-1}+3 \Psi_{i}+\Psi_{i+1}\right)
$$

\section{References}

[1] A.A. Mohamad, Lattice Boltzmann Method: Fundamentals and Engineering Applications with Computer Codes, Springer Science \& Business Media, 2011.

[2] Z. Guo, C. Shu, Lattice Boltzmann Method and Its Applications in Engineering, vol. 3, World Scientific, 2013.

[3] L. Chen, Q. Kang, Y. Mu, Y.-L. He, W.-Q. Tao, A critical review of the pseudopotential multiphase lattice Boltzmann model: methods and applications, Int. J. Heat Mass Transf. 76 (2014) 210-236.

[4] T. Krüger, H. Kusumaatmaja, A. Kuzmin, O. Shardt, G. Silva, E.M. Viggen, The Lattice Boltzmann Method, vol. 10, Springer International Publishing, 2017, p. 978.

[5] S. Succi, G. Amati, M. Bernaschi, G. Falcucci, M. Lauricella, A. Montessori, Towards exascale lattice Boltzmann computing, Comput. Fluids 181 (2019) $107-115$.

[6] Y. Feng, P. Boivin, J. Jacob, P. Sagaut, Hybrid recursive regularized lattice Boltzmann simulation of humid air with application to meteorological flows, Phys. Rev. E 100 (2) (2019) 023304.

[7] W. Shi, W. Shyy, R. Mei, Finite-difference-based lattice Boltzmann method for inviscid compressible flows, Numer. Heat Transf., Part B, Fundam. 40 (1) (2001) $1-21$

[8] T. Kataoka, M. Tsutahara, Lattice Boltzmann model for the compressible Navier-Stokes equations with flexible specific-heat ratio, Phys. Rev. E 69 (3) (2004) 035701.

[9] Q. Li, Y. He, Y. Wang, W. Tao, Coupled double-distribution-function lattice Boltzmann method for the compressible Navier-Stokes equations, Phys. Rev. E 76 (5) (2007) 056705.

[10] R. So, R. Leung, S. Fu, Modeled Boltzmann equation and its application to shock-capturing simulation, AIAA J. 46 (12) (2008) 3038-3048.

[11] Z. Guo, R. Wang, K. Xu, Discrete unified gas kinetic scheme for all Knudsen number flows. II. Thermal compressible case, Phys. Rev. E 91 (3) (2015) 033313.

[12] L. Yang, C. Shu, J. Wu, A hybrid lattice Boltzmann flux solver for simulation of viscous compressible flows, Adv. Appl. Math. Mech. 8 (6) (2016) 887-910.

[13] K. Xu, A gas-kinetic bgk scheme for the Navier-Stokes equations and its connection with artificial dissipation and Godunov method, J. Comput. Phys. 171 (1) (2001) 289-335.

[14] K. Xu, J.-C. Huang, A unified gas-kinetic scheme for continuum and rarefied flows, J. Comput. Phys. 229 (20) (2010) $7747-7764$.

[15] L. Yang, C. Shu, W. Yang, J. Wu, An improved three-dimensional implicit discrete velocity method on unstructured meshes for all Knudsen number flows, J. Comput. Phys. 396 (2019) 738-760.

[16] C. Liu, Y. Zhu, K. Xu, Unified gas-kinetic wave-particle methods i: continuum and rarefied gas flow, J. Comput. Phys. 401 (2020) 108977.

[17] P.J. Dellar, An interpretation and derivation of the lattice Boltzmann method using Strang splitting, Comput. Math. Appl. 65 (2013) $129-141$.

[18] F.J. Alexander, H. Chen, S. Chen, G. Doolen, Lattice Boltzmann model for compressible fluids, Phys. Rev. A 46 (4) (1992) 1967.

[19] X.W. Shan, X.F. Yuan, H.D. Chen, Kinetic theory representation of hydrodynamics: a way beyond the Navier-Stokes equation, J. Fluid Mech. 550 (2006) 413-441.

[20] A. Scagliarini, L. Biferale, M. Sbragaglia, K. Sugiyama, F. Toschi, Lattice Boltzmann methods for thermal flows: continuum limit and applications to compressible Rayleigh-Taylor systems, Phys. Fluids 22 (5) (2010) 055101.

[21] P. Philippi, D. Siebert, L. Hegele Jr, K. Mattila, High-order lattice-Boltzmann, J. Braz. Soc. Mech. Sci. Eng. 38 (5) (2016) 1401-1419.

[22] N. Frapolli, S.S. Chikatamarla, I.V. Karlin, Entropic lattice Boltzmann model for compressible flows, Phys. Rev. E 92 (6) (2015) 061301.

[23] X. Shan, Central-moment-based galilean-invariant multiple-relaxation-time collision model, Phys. Rev. E 100 (2019) 043308.

[24] N. Frapolli, Entropic lattice Boltzmann models for thermal and compressible flows, Ph.D. thesis, ETH, Zurich, 2017. 
[25] C. Coreixas, G. Wissocq, G. Puigt, J. Boussuge, P. Sagaut, Recursive regularization step for high-order lattice Boltzmann methods, Phys. Rev. E 96 (2017) 033306.

[26] K.K. Mattila, P.C. Philippi, L.A. Hegele Jr, High-order regularization in lattice-Boltzmann equations, Phys. Fluids 29 (4) (2017) 046103.

[27] M. Atif, M. Namburi, S. Ansumali, High-order lattice-Boltzmann model for thermodynamics, Phys. Rev. E 98 (2018) 053311.

[28] R. Qiu, R. Chen, C. Zhu, Y. You, A Hermite-based lattice Boltzmann model with artificial viscosity for compressible viscous flows, Int. J. Mod. Phys. B 32 (2018) 1850157.

[29] Y. Gan, A. Xu, G. Zhang, Y. Zhang, S. Succi, Discrete Boltzmann trans-scale modeling for high-speed compressible flows, Phys. Rev. E 97 (2018) 053312.

[30] L. Fei, K.H. Luo, Cascaded lattice Boltzmann method for thermal flows on standard lattices, Int. J. Therm. Sci. 132 (2018) 368-377.

[31] Y. Feng, P. Sagaut, W.Q. Tao, A compressible lattice Boltzmann finite volume model for high subsonic and transonic flows on regular lattices, Comput. Fluids 131 (2016) 45-55.

[32] M.H. Saadat, F. Bösch, I.V. Karlin, Lattice Boltzmann model for compressible flows on standard lattices: variable Prandtl number and adiabatic exponent, Phys. Rev. E 99 (1) (2019) 013306.

[33] J. Buick, J. Cosgrove, Investigation of a lattice Boltzmann model with a variable speed of sound, J. Phys. A, Math. Gen. 39 (44) (2006) 13807.

[34] X. Nie, X. Shan, H. Chen, Lattice Boltzmann/finite-difference hybrid simulation of transonic flow, AIAA Pap. 139 (2009) 2009.

[35] G. Romani, D. Casalino, Rotorcraft blade-vortex interaction noise prediction using the lattice-Boltzmann method, Aerosp. Sci. Technol. 88 (2019) $147-157$.

[36] Y. Feng, P. Sagaut, W. Tao, A three dimensional lattice model for thermal compressible flow on standard lattices, J. Comput. Phys. 303 (2015) $514-529$.

[37] J. Jacob, O. Malaspinas, P. Sagaut, A new hybrid recursive regularised Bhatnagar-Gross-Krook collision model for lattice Boltzmann method-based large eddy simulation, J. Turbul. 19 (11-12) (2018) 1051-1076.

[38] Y.-L. Feng, S.-L. Guo, W.-Q. Tao, P. Sagaut, Regularized thermal lattice Boltzmann method for natural convection with large temperature differences, Int. J. Heat Mass Transf. 125 (2018) 1379-1391.

[39] Y. Feng, M. Tayyab, P. Boivin, A lattice-Boltzmann model for low-Mach reactive flows, Combust. Flame 196 (2018) 249-254.

[40] Y. Feng, P. Boivin, J. Jacob, P. Sagaut, Hybrid recursive regularized thermal lattice Boltzmann model for high subsonic compressible flows, J. Comput. Phys. 394 (2019) 82-99.

[41] F. Renard, Y. Feng, J. Boussuge, P. Sagaut, Improved compressible hybrid lattice Boltzmann method on standard lattice for subsonic and supersonic flows, Comput. Fluids (2020), submitted for publication, arXiv:2002.03644.

[42] H. Yu, K. Zhao, Lattice Boltzmann method for compressible flows with high Mach numbers, Phys. Rev. E 61 (4) (2000) 3867.

[43] G. Yan, J. Zhang, Y. Liu, Y. Dong, A multi-energy-level lattice Boltzmann model for the compressible Navier-Stokes equations, Int. J. Numer. Methods Fluids 55 (1) (2007) 41-56.

[44] N.I. Prasianakis, I.V. Karlin, Lattice Boltzmann method for simulation of compressible flows on standard lattices, Phys. Rev. E 78 (1) (2008) 016704.

[45] Q. Li, K. Luo, Y. He, W. Tao, Coupling lattice Boltzmann model for simulation of thermal flows on standard lattices, Phys. Rev. E 85 (2012) 016710.

[46] X. Li, Y. Shi, X. Shan, et al., Temperature-scaled collision process for the high-order lattice Boltzmann model, Phys. Rev. E 100 (1) (2019) 013301.

[47] M.H. Saadat, F. Bösch, I.V. Karlin, Lattice Boltzmann model for compressible flows on standard lattices: variable Prandtl number and adiabatic exponent, Phys. Rev. E 99 (1) (2019) 013306.

[48] Y. Chen, H. Ohashi, M. Akiyama, Thermal lattice Bhatnagar-Gross-Krook model without nonlinear deviations in macrodynamic equations, Phys. Rev. E 50 (4) (1994) 2776.

[49] G.R. McNamara, A.L. Garcia, B.J. Alder, A hydrodynamically correct thermal lattice Boltzmann model, J. Stat. Phys. 87 (5-6) (1997) $1111-1121$.

[50] Y. Li, A. Jammalamadaka, P. Gopalakrishnan, R. Zhang, H. Chen, Computation of high-subsonic and transonic flows by a lattice Boltzmann method, in: 54th AIAA Aerospace Sciences Meeting, 2016, p. 0043.

[51] X. Shan, The mathematical structure of the lattices of the lattice Boltzmann method, J. Comput. Sci. 17 (2016) 475-481.

[52] E. Fares, M. Wessels, R. Zhang, C. Sun, N. Gopalaswamy, P. Roberts, J. Hoch, H. Chen, Validation of a lattice-Boltzmann approach for transonic and supersonic flow simulations, in: 52nd Aerospace Sciences Meeting, 2014, p. 0952.

[53] D. Singh, B. Konig, E. Fares, M. Murayama, Y. Ito, Y. Yokokawa, K. Yamamoto, Lattice-Boltzmann simulations of the jaxa jsm high-lift configuration in a wind tunnel, in: AIAA Scitech 2019 Forum, 2019, p. 1333.

[54] J. Latt, C. Coreixas, J. Beny, A. Parmigiani, Efficient supersonic flows through high-order guided equilibrium with lattice Boltzmann, arXiv preprint arXiv:1910.13515, 2019

[55] B. Yang, J. Flusser, T. Suk, 3d rotation invariants of Gaussian-Hermite moments, Pattern Recognit. Lett. 54 (2015) 18-26.

[56] O. Malaspinas, Increasing stability and accuracy of the lattice Boltzmann scheme: recursivity and regularization, arXiv preprint arXiv:1505.06900.

[57] A.E. Honein, P. Moin, Higher entropy conservation and numerical stability of compressible turbulence simulations, J. Comput. Phys. 201 (2) (2004) 531-545.

[58] V. Levasseur, P. Sagaut, F. Chalot, A. Davroux, An entropy-variable-based vms/gls method for the simulation of compressible flows on unstructured grids, Comput. Methods Appl. Mech. Eng. 195 (9-12) (2006) 1154-1179.

[59] Y. Kuya, K. Totani, S. Kawai, Kinetic energy and entropy preserving schemes for compressible flows by split convective forms, J. Comput. Phys. 375 (2018) 823-853.

[60] G. Coppola, F. Capuano, S. Pirozzoli, L. de Luca, Numerically stable formulations of convective terms for turbulent compressible flows, J. Comput. Phys. 382 (2019) 86-104.

[61] A. Jameson, Formulation of kinetic energy preserving conservative schemes for gas dynamics and direct numerical simulation of one-dimensional viscous compressible flow in a shock tube using entropy and kinetic energy preserving schemes, J. Sci. Comput. 34 (2008) 188-208.

[62] A. Jameson, The construction of discretely conservative finite volume schemes that also globally conserve energy of entropy, J. Sci. Comput. 34 (2008) $152-187$.

[63] Y. Abe, I. Morinaka, T. Haga, T. Nonomura, H. Shibita, K. Miyaji, Stable, non-dissipative, and conservative flux-reconstruction schemes in split forms, J. Comput. Phys. 353 (2018) 193-227.

[64] P. Chandrashekar, Kinetic energy preserving and entropy stable finite volume schemes for compressible Euler and Navier-Stokes equations, Commun. Comput. Phys. 14 (2013) 1252-1286.

[65] P. Ma, Y. Lv, M. Ihme, An entropy-stable hybrid scheme for simulations of trans-critical real-fluid flows, J. Comput. Phys. 340 (2017) $330-357$.

[66] J. Chan, On discretely entropy conservative and entropy stable discontinuous Galerkin methods, J. Comput. Phys. 362 (2018) 346-374.

[67] N. Frapolli, S. Chikatamarla, I. Karlin, Entropic lattice Boltzmann simulation of thermal convective turbulence, Comput. Fluids 175 (2018) 2-19.

[68] N. Frapolli, S. Chikatamarla, I. Karlin, Multispeed entropic lattice Boltzmann model for thermal flows, Phys. Rev. E 90 (4) (2014) 043306.

[69] N.I. Prasianakis, S.S. Chikatamarla, I.V. Karlin, S. Ansumali, K. Boulouchos, Entropic lattice Boltzmann method for simulation of thermal flows, Math. Comput. Simul. 72 (2006) 179-183.

[70] Y.-H. Qian, Y. Zhou, Higher-order dynamics in lattice-based models using the Chapman-Enskog method, Phys. Rev. E 61 (2) (2000) 2103.

[71] K.H. Kim, C. Kim, O.-H. Rho, Methods for the accurate computations of hypersonic flows: I. AUSMPW+ scheme, J. Comput. Phys. 174 (1) (2001) 38-80.

[72] C. Hirsch, Numerical Computation of Internal and External Flows: The Fundamentals of Computational Fluid Dynamics, Elsevier, 2007.

[73] A. Jameson, Origins and further development of the Jameson-Schmidt-Turkel scheme, AIAA J. (2017) 1487-1510. 
[74] Y. Morinishi, T. Lund, O. Vasilyev, P. Moin, Fully conservative higher order finite difference schemes for incompressible flows, J. Comput. Phys. 143 (1998) 90-124.

[75] M. Droge, R. Verstappen, A new symmetry-preserving Cartesian-grid for computing flow pas arbitrarily shaped objects, Int. J. Numer. Methods Fluids 47 (2005) 979-985.

[76] Y. Morinishi, Skew-symmetric form of convective terms and fully conservative finite difference schemes for variable density low-Mach number flows, J. Comput. Phys. 229 (2010) 1276-1300.

[77] S. Pirozzoli, Stabilized non-dissipative approximations of Euler equations in generalized curvilinear coordinates, J. Comput. Phys. 230 (2011) $2997-3014$.

[78] Y. Morinishi, K. Koga, Skew-symmetric convection form and secondary conservative finite difference methods for moving grids, J. Comput. Phys. 257 (2014) 1081-1112.

[79] F. Capuano, G. Coppola, G. Balarac, L. de Luca, Energy preserving turbulent simulations at a reduced computational cost, J. Comput. Phys. 298 (2015) 480-494.

[80] F. Capuano, G. Coppola, L. de Luca, An efficient time advancing strategy for energy-preserving simulations, J. Comput. Phys. 295 (2015) $209-229$.

[81] J. Latt, B. Chopard, Lattice Boltzmann method with regularized pre-collision distribution functions, Math. Comput. Simul. 72 (2006) 165-168.

[82] ProLB: high-fidelity CFD in exceptional turnaround times, http://www.prolb-cfd.com, 2019.

[83] S. Marié, D. Ricot, P. Sagaut, Comparison between lattice Boltzmann method and Navier-Stokes high order schemes for computational aeroacoustics, J. Comput. Phys. 228 (4) (2009) 1056-1070.

[84] M. Dumbser, E.F. Toro, On universal Osher-type schemes for general nonlinear hyperbolic conservation laws, Commun. Comput. Phys. 10 (3) (2011) 635-671.

[85] M. Tavelli, M. Dumbser, A pressure-based semi-implicit space-time discontinuous Galerkin method on staggered unstructured meshes for the solution of the compressible Navier-Stokes equations at all Mach numbers, J. Comput. Phys. 341 (2017) 341-376.

[86] M.H. Djavareshkian, M.A. Jahdi, Shock-capturing method using characteristic-based dissipation filters in pressure-based algorithm, Acta Mech. 209 (1-2) (2010) 99.

[87] Amroc-blockstructured adaptive mesh refinement in object-oriented c++, http://amroc.sourceforge.net/examples/euler/3d/html/pbc3d_vis.htm, 2019.

[88] O. Inoue, Y. Hattori, Sound generation by Shock-Vortex interactions, J. Fluid Mech. 380 (1999) 81-116.

[89] D.R. Chapman, Temperature and velocity profiles in the compressible laminar boundary layer with arbitrary distribution of surface temperature, J. Aeronaut. Sci. 16 (9) (1949) 547-565.

[90] D. De Grazia, D. Moxey, S. Sherwin, M. Kravtsova, A. Ruban, Direct numerical simulation of a compressible boundary-layer flow past an isolated three-dimensional hump in a high-speed subsonic regime, Phys. Rev. Fluids 3 (2) (2018) 024101.

[91] F.M. White, I. Corfield, Viscous Fluid Flow, vol. 3, McGraw-Hill, New York, 2006.

[92] T.Y. Hou, P.G. Lefloch, Why nonconservative schemes converge to wrong solutions: error analysis, Math. Comput. 62 (206) (1994) 497-530.

[93] R. Abgrall, S. Karni, A comment on the computation of non-conservative products, J. Comput. Phys. 229 (8) (2010) 2759-2763.

[94] R. Abgrall, P. Bacigaluppi, S. Tokareva, A high-order nonconservative approach for hyperbolic equations in fluid dynamics, Comput. Fluids 169 (2018) $10-22$.

[95] L. Gastaldo, R. Herbin, J.-C. Latché, N. Therme, A MUSCL-type segregated - explicit staggered scheme for the Euler equations, Comput. Fluids 175 (2018) $91-110$.

[96] K. Xu, Regularization of the Chapman-Enskog expansion and its description of shock structure, Phys. Fluids 14 (4) (2002) L17-L20.

[97] H. Struchtrup, M. Torrilhon, Regularization of Grad's 13 moment equations: derivation and linear analysis, Phys. Fluids 15 (9) (2003) $2668-2680$. 